\title{
A POD Reduced Order Model for Resolving Angular Direction in Neutron/Photon Transport Problems
}

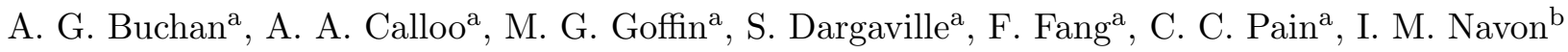 \\ ${ }^{a}$ Applied Modelling and Computation Group \\ Department of Earth Science and Engineering \\ Imperial College London, SW7 2AZ, UK \\ ${ }^{b}$ Department of Scientific Computing \\ Florida State University, Tallahassee, FL 32306-4120, USA
}

\begin{abstract}
This article presents the first Reduced Order Model (ROM) that efficiently resolves the angular dimension of the time independent, mono-energetic Boltzmann Transport Equation (BTE). It is based on Proper Orthogonal Decomposition (POD) and uses the method of snapshots to form optimal basis functions for resolving the direction of particle travel in neutron/photon transport problems. A unique element of this work is that the snapshots are formed from the vector of angular coefficients relating to a high resolution expansion of the BTE's angular dimension. In addition, the individual snapshots are not recorded through time, as in standard POD, but instead they are recorded through space. In essence this work swaps the roles of the dimensions space and time in standard POD methods, with angle and space respectively.
\end{abstract}

It is shown here how the POD model can be formed from the POD basis functions in a highly efficient manner. The model is then applied to two radiation problems; one involving the transport of radiation through a shield and the other through an infinite array of pins. Both problems are selected for their complex angular flux solutions in order to provide an appropriate demonstration of the model's capabilities. It is shown that the POD model can resolve these fluxes efficiently and accurately. In comparison to high resolution models this POD model can reduce the size of a problem

Email address: andrew.buchan@imperial.ac.uk (A. G. Buchan) 
by up to two orders of magnitude without compromising accuracy. Solving times are also reduced by similar factors.

Keywords: Neutron Transport; Photon Transport; Boltzmann Transport Equation; Reduced Order Model; Proper Orthogonal Decomposition

\section{Introduction}

Computational models are important tools for our understanding of many fields of physics, they are relied upon to predict, safeguard, and optimise designs used throughout industry and academia. However despite today's large computational resources, numerical methods used to discretise the underlying equations can still require excessive computing times. Complex domains, high detail solutions or equations with high dimensionality can quickly lead to models using a large number of degrees of freedom. Solving such systems can require high computing effort, and as a direct result in reducing this burden, Reduce Order Methods (ROMs) have evolved. These are techniques that efficiently resolve problems through reduced dimensionality. One such method, which has gained much popularity in recent years, is that of Proper Orthogonal Decomposition.

Proper orthogonal decomposition has evolved under a number of names from various fields. It is known as Karhunen-Loéve expansions in signal analysis and pattern recognition [1], principal component analysis in statistics [2], the method of empirical orthogonal functions in geophysical fluid dynamics [3] and meteorology [4]. All of these are model reduction techniques that offer efficient approximations of dynamical systems through using a reduced number of degrees of freedom $[5,6,7]$. The fundamental mechanics of POD are to generate optimal basis functions that represent and capture the energy, or dynamics, of a system of interest, and a way of achieving this is through the method of snapshots [8]. This involves taking snapshots of the system's state at various time instances [9], and forming optimal basis functions for their representation.

The origins of POD dates back some way to the early 1900's in the work of Pearson [10], but following the pioneering work of Lumley [11] it has received a considerable amount of attention from within the fluid dynamics community. Early applications include the work of Bakewell [12] and Payne [13] who respectively applied the techniques in turbulent pipe flow and wakes behind cylinders. Other applications include the modelling of flows around air foils [14] and through channels [15], the mixing of fluid layers [16], thermal currents [17, 18] and ocean models [19]. It has been applied to the shallow water equations [20], the Euler equations [21], the full Navier Stokes equations [22] and the various 
reduced versions of it including the parabolized Navier Stokes equation [23, 24]. POD has been applied to many other fields which are covered extensively in the references of the articles listed here.

In this article the method of POD is applied in a very unique way to solve radiation transport problems. It is applied to the Boltzmann Transport Equation (BTE), which describes the transport of radiation, in order to reduce the problem of high dimensionality (which plagues many radiation transport (RT) models). The problem of high dimensionality arises from several factors, one of the main being the equation having a 7 dimensional phase-space (their discretisation lead to high numbers of unknowns). Radiation problems are also made difficult by their geometries being complex (e.g. reactors are formed of thousands of pins and shields have complex duct systems) and solutions requiring high resolution. POD can therefore be of benefit to these radiation problems as it possesses the appropriate properties to efficiently resolve them. In addition, the BTE is a linear equation which side-steps completely the issue of non-efficient operations caused by non-linear operators. This means that additional treatment of the non-linear terms, recent examples including those of quadratic expansion [25], DEIM [26] and Residual-DEIM [27], are not necessary here.

The novelty in the method presented here is in the use of POD to resolve the direction of particle travel. The two angular dimensions of the BTE are resolved using POD basis functions formed from the method of snapshots. Unusually the snapshots are taken through space, rather than the traditional time, and this in turn allows for time-independent problems to be solved (although it should be mentioned that time dependent problems can still be solved). This has similarities with that of $[23,24]$, which solves the two dimensional time independent parabolized Navier-Stokes equation using a spatial dimension as though it were time. The work here shows how the POD model can be constructed efficiently. It is also shown how the same full model methods can be used to resolve the remaining dimensions of the BTE. This is particularly important as it makes the implementation of the POD model simple as it is almost non-intrusive.

The application of ROMs to radiation problems is still quite rare, although there has been some development in recent years. In [28] POD models have been applied to simulate the dynamics of an accelerator driven system (ADS), and in [29] a useful comparison of POD and modal methods were made. In the work of [30] POD was applied to solving eigenvalue problems for reactor physics applications. The work of [31] applied a Karhunen-Loéve approach to form basis functions that efficiently resolve the energy dependence in reactor physics problems.

The sections of this article are set out as follows. In section 2 the Boltzmann transport equation is introduced and a description of the high resolution discretisation of the space and angle dimensions is given. This section then describes the process of recording the snapshot data and forming the new 
reduced order model. In section 3 two numerical examples are presented. These are specifically chosen to pose complex problems for the POD model to resolve, they also form realistic radiation transport problems. Section 4 completes this article with a conclusion of its findings.

\section{The POD formulation for Angular Discretisation}

The following sections describe transport equation together with a review of the full model's discretisation methods. Following this the formulation of the POD model is presented.

\subsection{The Boltzmann Transport Equation}

In this article the steady-state, mono-energetic Boltzmann transport equation is considered,

$$
\hat{\mathbf{\Omega}} \cdot \nabla \psi(\boldsymbol{r}, \hat{\mathbf{\Omega}})+\Sigma_{t}(\boldsymbol{r}) \psi(\boldsymbol{r}, \hat{\mathbf{\Omega}})=\int_{\hat{\mathbf{\Omega}}^{\prime}} \mathrm{d} \boldsymbol{\Omega}^{\prime} \Sigma_{s}\left(\boldsymbol{r}, \hat{\mathbf{\Omega}}^{\prime} \rightarrow \hat{\mathbf{\Omega}}\right) \psi\left(\boldsymbol{r}, \hat{\mathbf{\Omega}}^{\prime}\right)+S(\boldsymbol{r}, \hat{\mathbf{\Omega}}) .
$$

This is a linear equation but with five-dimensions (as energy and time are omitted) that describes the state of the angular flux $\psi$ in term of the spatial position, $\boldsymbol{r}$, and the angular direction, $\hat{\boldsymbol{\Omega}}$. The angular flux defines the number of neutral particles at position $\boldsymbol{r}$ travelling in direction $\hat{\boldsymbol{\Omega}}$ multiplied by their velocity. The terms in the equation define the change in flux due to particle streaming, absorption, scattering and external sources, respectively. The focus of this article is on the discretisation of the angular dimension of this equation. This is formed of a polar angle, $\theta$ (which is also expressed in the form of $\mu=\cos (\theta)$ ), and the azimuthal angle, $\omega$, as is illustrated in figure 1. The angle $\hat{\boldsymbol{\Omega}}$ can also be expressed in Cartesian coordinates $\hat{\Omega}=\left(\hat{\Omega}_{x}, \hat{\Omega}_{y}, \hat{\Omega}_{z}\right)$, which is the projection of the angle in the $\mathrm{x}, \mathrm{y}, \mathrm{z}$ plane. The components of this angular vector can be expressed as,

$$
\begin{gathered}
\hat{\Omega}_{z}=\mu=\cos \theta, \\
\hat{\Omega}_{x}=\left(1-\mu^{2}\right)^{\frac{1}{2}} \cos \omega, \\
\hat{\Omega}_{y}=\left(1-\mu^{2}\right)^{\frac{1}{2}} \sin \omega .
\end{gathered}
$$

Two boundary conditions applied in this article are those of vacuum and reflection. For a point $\boldsymbol{r}$ on the boundary with outward pointing normal $\boldsymbol{n}$, a vacuum condition requires that no particles enter the domain, i.e.,

$$
\psi(\boldsymbol{r}, \hat{\mathbf{\Omega}})=0, \text { where } \hat{\mathbf{\Omega}} \cdot \boldsymbol{n}<0 .
$$




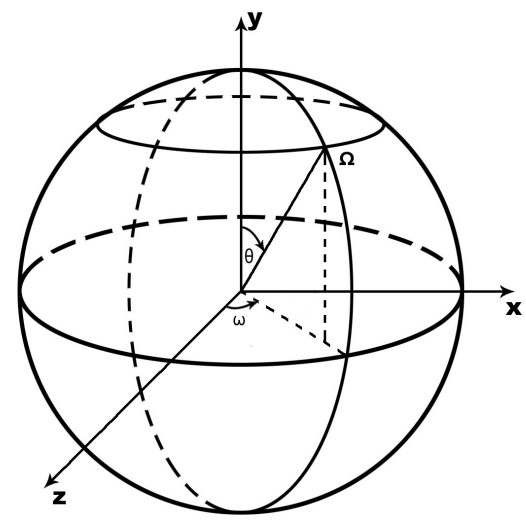

Figure 1: Spherical coordinates on the unit sphere, the polar coordinate $\mu=\cos (\theta)$.

For specular reflection the condition

$$
\psi(\boldsymbol{r}, \hat{\mathbf{\Omega}})=\psi\left(\boldsymbol{r}, \hat{\mathbf{\Omega}}^{*}\right), \text { where } \hat{\boldsymbol{\Omega}} \cdot \boldsymbol{n}<0,
$$

is required to hold where $\hat{\boldsymbol{\Omega}}^{*}$ is the reflection of $\hat{\boldsymbol{\Omega}}$ with respect to $\boldsymbol{n}$.

\subsection{The Angular Discretised Transport Equation}

In the derivation of the full model used in this article, equation 1 is first approximated in angle. The angular flux is expanded through a summation of $n_{m}$ angular basis functions, $G_{j}(\hat{\mathbf{\Omega}})$, for $j \in$ $\left\{1,2,3, \ldots, n_{m}\right\}$. This is given as,

$$
\psi(\boldsymbol{r}, \hat{\boldsymbol{\Omega}}) \approx \sum_{j=1}^{n_{m}} G_{j}(\hat{\boldsymbol{\Omega}}) \Psi_{j}(\boldsymbol{r}),
$$

where $\Psi_{i}(\boldsymbol{r})$ are the expansion coefficients. This expansion is substituted into equation 1 and a Galerkin projection applied whereby the the equation is weighted with the angular basis function 
$G_{i}(\hat{\mathbf{\Omega}})$, for $i \in\left\{1,2, \ldots, n_{m}\right\}$, and integrated over angle.

$$
\begin{aligned}
& \int_{\boldsymbol{\Omega}} G_{i}(\hat{\boldsymbol{\Omega}}) \Omega_{x} \frac{\partial}{\partial x}\left(\sum_{j=1}^{n_{m}} \Psi_{j}(\boldsymbol{r}) G_{j}(\hat{\boldsymbol{\Omega}})\right) \mathrm{d} \boldsymbol{\Omega}+\int_{\boldsymbol{\Omega}} G_{i}(\hat{\boldsymbol{\Omega}}) \Omega_{y} \frac{\partial}{\partial y}\left(\sum_{j=1}^{n_{m}} \Psi_{j}(\boldsymbol{r}) G_{j}(\hat{\boldsymbol{\Omega}})\right) \mathrm{d} \boldsymbol{\Omega} \\
& +\int_{\boldsymbol{\Omega}} G_{i}(\hat{\boldsymbol{\Omega}}) \Omega_{z} \frac{\partial}{\partial z}\left(\sum_{j=1}^{n_{m}} \Psi_{j}(\boldsymbol{r}) G_{j}(\hat{\boldsymbol{\Omega}})\right) \mathrm{d} \boldsymbol{\Omega}+\int_{\boldsymbol{\Omega}} G_{i}(\hat{\boldsymbol{\Omega}}) \Sigma_{t}(\boldsymbol{r})\left(\sum_{j=1}^{n_{m}} \Psi_{j}(\boldsymbol{r}) G_{j}(\hat{\boldsymbol{\Omega}})\right) \mathrm{d} \boldsymbol{\Omega} \\
& =\int_{\boldsymbol{\Omega}} G_{i}(\hat{\boldsymbol{\Omega}}) \int_{\hat{\boldsymbol{\Omega}}^{\prime}} \Sigma_{s}\left(\boldsymbol{r}, \hat{\boldsymbol{\Omega}}^{\prime} \rightarrow \boldsymbol{\Omega}\right)\left(\sum_{j=1}^{n_{m}} \Psi_{j}(\boldsymbol{r}) G_{j}\left(\hat{\boldsymbol{\Omega}}^{\prime}\right)\right) \mathrm{d} \boldsymbol{\Omega}^{\prime} \mathrm{d} \boldsymbol{\Omega}+\int_{\boldsymbol{\Omega}} G_{i}(\hat{\boldsymbol{\Omega}}) S(\boldsymbol{r}, \hat{\boldsymbol{\Omega}}) \mathrm{d} \boldsymbol{\Omega} .
\end{aligned}
$$

With a little rearranging, equation 4 can then be shown to give,

$$
\begin{gathered}
\sum_{j=1}^{n_{m}}\left[\int_{\boldsymbol{\Omega}} G_{i}(\Omega) \Omega_{x} G_{j}(\Omega) \mathrm{d} \boldsymbol{\Omega}\right] \frac{\partial}{\partial x} \Psi_{j}(\boldsymbol{r})+\sum_{j=1}^{n_{m}}\left[\int_{\boldsymbol{\Omega}} G_{i}(\hat{\boldsymbol{\Omega}}) \Omega_{y} G_{j}(\hat{\boldsymbol{\Omega}}) \mathrm{d} \boldsymbol{\Omega}\right] \frac{\partial}{\partial y} \Psi_{j}(\boldsymbol{r}) \\
+\sum_{j=1}^{n_{m}}\left[\int_{\boldsymbol{\Omega}} G_{i}(\hat{\boldsymbol{\Omega}}) \Omega_{z} G_{j}(\hat{\boldsymbol{\Omega}}) \mathrm{d} \boldsymbol{\Omega}\right] \frac{\partial}{\partial z} \Psi_{j}(\boldsymbol{r})+\sum_{j=1}^{n_{m}}\left[\int_{\boldsymbol{\Omega}} G_{i}(\hat{\boldsymbol{\Omega}}) \Sigma_{t}(\boldsymbol{r}) G_{j}(\hat{\boldsymbol{\Omega}}) \mathrm{d} \boldsymbol{\Omega}\right. \\
\left.-\int_{\boldsymbol{\Omega}} G_{i}(\boldsymbol{\Omega}) \int_{\boldsymbol{\Omega}^{\prime}} \Sigma_{s}\left(\boldsymbol{r}, \hat{\boldsymbol{\Omega}}^{\prime} \rightarrow \hat{\boldsymbol{\Omega}}\right) G_{j}\left(\hat{\boldsymbol{\Omega}}^{\prime}\right) \mathrm{d} \boldsymbol{\Omega}^{\prime} \mathrm{d} \boldsymbol{\Omega}\right] \Psi_{j}(\boldsymbol{r})=\left[\int_{\boldsymbol{\Omega}} G_{i}(\hat{\boldsymbol{\Omega}}) S(\boldsymbol{r}, \hat{\boldsymbol{\Omega}}) \mathrm{d} \boldsymbol{\Omega}\right] \forall i \in\left\{1,2, \ldots, n_{m}\right\} .
\end{gathered}
$$

which can be condensed into the following matrix form,

$$
A_{x} \frac{\partial \boldsymbol{\Psi}}{\partial x}+A_{y} \frac{\partial \boldsymbol{\Psi}}{\partial y}+A_{z} \frac{\partial \boldsymbol{\Psi}}{\partial z}+H \boldsymbol{\Psi}=S .
$$

This system defines the angular discretised equations where the vector $\Psi(\boldsymbol{r})$ denotes the vector of $n_{m}$ angular coefficients of equation 3. Terms $A_{x}, A_{y}$ and $A_{z}$ are $n_{m}$ by $n_{m}$ matrices that represent the angular discretised streaming term, while $H$ is an $n_{m}$ by $n_{m}$ matrix containing the discretised scattering-removal terms. The term $S$ denoted the angularly discretised source, which is a vector of length $n_{m}$. For full details in computing these terms see [32].

\subsection{The Spatial Discretisation}

Here a general finite element discretisation of the spatial variable $\boldsymbol{r}$ is presented. Whilst the numerical examples presented in this article use a sub-grid scale formulation [33, 34], it is sufficient to consider a simple Bubnov Galerkin projection of the spatial domain using linear finite elements. The spatial 
dependence of the angularly discretised flux in equation 6 is represented as an expansion of finite element (FE) functions,

$$
\Psi(\boldsymbol{r}) \approx \sum_{l}^{n_{s}} N_{l}(\boldsymbol{r}) \mathbf{\Psi}_{l}
$$

The terms $N_{l}$ represent a diagonal matrix of size $n_{m} \times n_{m}$ containing the $l^{\text {th }}$ finite element function. The terms $\boldsymbol{\Psi}_{l}$ are vectors of size $n_{m}$ containing the unknown angular moments associated with the finite element functions of $N_{l}$. The Galerkin projection of the angular discretised transport equation reads as,

$$
\int_{V} N_{k}\left((\boldsymbol{A} \cdot \boldsymbol{\nabla}+H(\boldsymbol{r})) \sum_{l}^{n_{s}} N_{l}(\boldsymbol{r}) \boldsymbol{\Psi}_{l}-\boldsymbol{S}(\boldsymbol{r})\right) \mathrm{d} \boldsymbol{V}=\mathbf{0}, \forall k \in\left\{1, \ldots, n_{s}\right\} .
$$

If the external angular discretised source is also represented as an expansion of FE basis functions, equation 8 can be written as,

$$
\int_{\boldsymbol{V}} N_{k} H \sum_{l}^{n_{s}} N_{l}(\boldsymbol{r}) \boldsymbol{\Psi}_{l} \mathrm{~d} \boldsymbol{V}+\int_{\boldsymbol{V}} N_{k} \boldsymbol{\nabla} \cdot \boldsymbol{A} \sum_{l}^{n_{s}} N_{l}(\boldsymbol{r}) \boldsymbol{\Psi}_{l} \mathrm{~d} \boldsymbol{V}=\int_{\boldsymbol{V}} N_{k} \sum_{l}^{n_{s}} N_{l}(\boldsymbol{r}) \boldsymbol{S}_{l}, \quad \forall k \in\left\{1, \ldots, n_{s}\right\} .
$$

Green's theory can now be applied to the advection term of this formulation to give,

$$
\begin{aligned}
\sum_{l}^{n_{s}} \int_{\boldsymbol{V}} N_{k} H N_{l}(\boldsymbol{r}) \mathrm{d} \boldsymbol{V} \boldsymbol{\Psi}_{l} & -\sum_{l}^{n_{s}} \int_{\boldsymbol{V}} \boldsymbol{\nabla} N_{k} \cdot \boldsymbol{A} N_{l} \mathrm{~d} \boldsymbol{V} \mathbf{\Psi}_{l}+ \\
\sum_{l}^{n_{s}} \int_{\boldsymbol{\Gamma}} N_{k}(\boldsymbol{A} \cdot \boldsymbol{n}) N_{l} \mathrm{~d} \boldsymbol{\Gamma} \boldsymbol{\Psi}_{l} & =\sum_{l}^{n_{s}} \int_{\boldsymbol{V}} N_{k} N_{l}(\boldsymbol{r}) \mathrm{d} \boldsymbol{V} \boldsymbol{S}_{l}, \quad \forall k \in\left\{1, \ldots, n_{s}\right\},
\end{aligned}
$$

where a surface integral forms across the problem's boundary $\boldsymbol{\Gamma}$. As previously mentioned the Bubnov Galerkin projection is sufficient to describe the finite element formulation of the POD model, as the four terms in equation 10 appear, in one form or another, in all Petrov-Galerkin/sub-grid scale formulations (these formulation are necessary to use in radiation modelling as they provide the stable non-oscillatory solutions that Bubnov Galerkin does not). That is, there are terms involving the streaming operators, the scattering-removal terms, boundary conditions and external sources. At this point it is important to mention the boundary term in this equation and describe how this is treated in order to satisfy conditions placed on the problem's surface. A Riemann approach [35] is used here that manipulates the matrix $(\boldsymbol{A} \cdot \boldsymbol{n})$ inside the surface integral. For any arbitrary angular approximation, this Riemann method separates the integral into two components containing the incoming and outgoing information. This enables the standard surface conditions to be satisfied, for example, void conditions can be removed by setting the incoming component to zero. The full details of the Riemann formulation are covered in $[33,35]$, however here it is sufficient to state the method results in a new surface integral,

$$
\sum_{l}^{n_{s}} \int_{\Gamma} N_{k} R_{n} N_{l} \mathrm{~d} \boldsymbol{\Gamma} \mathbf{\Psi}_{l}
$$


that replaces the surface integral in equation 10. The term $R_{n}$ is an $n_{m} \times n_{m}$ 'Riemann matrix' that forces the boundary condition to be satisfied. As previously mentioned, this method works for arbitrary angular discretisations and this is important for the POD model formulation.

\subsection{The POD Angular Discretisation}

The POD model discretisation of the angular dimension of the radiation transport equation is described in this section. The formulation of the model follows that of a standard POD approach, whereby POD basis functions are formed from the snapshots of solutions generated by a full, or high resolution, model. The full model used in this article employs a sub-grid scale finite element discretisation of the spatial domain and spherical harmonic expansions of the angular variable. A full discussion of these formulations can be found in $[33,36]$.

Standard POD formulations are commonly employed to solve time dependent problems whereby the POD basis functions are formed from the snapshots of solutions as they vary in time. The reduced order model described here differs from this traditional POD approach in a number of ways. The most significant of these differences is that this model is used to solve time independent radiation transport problems; the model is also used to form optimal basis functions for resolving angular direction. The snapshots used to form the POD basis functions are therefore vectors of angular moments, as defined in equation 6. As no time dimension is available in which to take the snapshots, the set of snapshots are formed by recording these moments at different positions in space - in essence this work has interchanged the roles of space and time in standard POD, with angle and space, respectively (for similar techniques see $[23,24])$. The snapshots of angular moments are taken at each node defined by the full model's mesh. That is, on each node $i$ of the FE mesh, the angular vector $\boldsymbol{\Psi}_{i}$ of equation 10 forms the $i^{t h}$ snapshot, and these are aligned in columns to form the snapshot matrix $\mathcal{A}$. The size of this matrix will be of size $n_{m} \times n_{s}$.

The POD basis functions are obtained through the singular value decomposition (SVD) of the matrix $\mathcal{A}$. In many POD formulations, the mean values of the snapshot matrix's rows are first removed before

the SVD is applied. However here the SVD is performed on the original matrix, the reasons for this will become clear shortly. The SVD of $\mathcal{A}$ is given by the expression

$$
\mathcal{A}=U \Sigma V^{T}
$$

where $U$ and $V$ are orthogonal matrices of dimensions $n_{m} \times n_{m}$ and $n_{s} \times n_{s}$ respectively. $\Sigma$ is a diagonal matrix of size $n_{m} \times n_{s}$ and contains the singular values of $\mathcal{A}$, denoted by $\sigma_{1}, \sigma_{2}, \ldots, \sigma_{n_{f}}$, where $n_{f}=\min \left\{n_{m}, n_{s}\right\}$. These singular values are arranged in $\Sigma$ in descending order. 
The POD basis vectors $\Phi_{j}$ that define the POD basis functions are defined by the columns of $U$.

$$
\Phi_{j}=U_{:, j} \forall j \in\left\{1,2, \ldots, n_{f}\right\} .
$$

From these POD vectors the POD basis functions can be formed by the following expression involving the full model's angular basis functions,

$$
\Phi_{j}(\boldsymbol{\Omega})=\sum_{i=1}^{n_{m}}\left(\Phi_{j}\right)_{i} G_{i}(\boldsymbol{\Omega})=\sum_{i=1}^{n_{m}} U_{i, j} G_{i}(\boldsymbol{\Omega}) \forall j \in\left\{1,2, \ldots, n_{f}\right\} .
$$

As with all POD methods, these basis functions are optimal in the sense that they provide the best basis functions that span the snapshot data. That is, for a given $n_{r}<n_{f}$, the first $n_{r}$ POD basis functions defined in equation 14 provide the optimal basis functions such that no other basis set of the same size can represent the data more accurately. This is equivalent to retaining the first $n_{r}$ columns of the matrix $U$ that correspond to the largest $n_{r}$ singular values of $\Sigma$, and reconstructing the matrix $\mathcal{A}$ using equation 12 . The resulting reconstruction will be the closest rank $n_{r}$ matrix, in the Frobenius norm, to the original snapshot matrix $\mathcal{A}$. A measurement of the information, or energy, of the snapshot data that is captured by $n_{r}$ POD basis functions is given by the expression,

$$
I=\frac{\sum_{i}^{n_{r}} \lambda_{i}}{\sum_{i}^{n_{f}} \lambda_{i}}
$$

where $\lambda_{i}$ are the squared values of the singular values $\sigma_{i}$. The value of $I$ will vary from 0 to 1 , the latter of which being the complete capture of the snapshot data.

The angular flux can now be expanded in angle in terms of the POD basis functions. In continuous form this reads as,

$$
\psi(\boldsymbol{r}, \hat{\mathbf{\Omega}})=\sum_{j=1}^{n_{r}} \Phi_{j}(\hat{\mathbf{\Omega}}) \alpha_{j}(\boldsymbol{r}),
$$

where $\alpha_{j}(\boldsymbol{r})$ denote the set of expansion coefficients. Alternatively in discrete form the approximation reads as,

$$
\boldsymbol{\Psi}(\boldsymbol{r})=\sum_{j=1}^{n_{r}} \Phi_{j} \alpha_{j}(\boldsymbol{r})=\sum_{j=1}^{n_{r}} \mathcal{U}_{:, j} \alpha_{j}(\boldsymbol{r})=\mathcal{U} \boldsymbol{\alpha}(\boldsymbol{r}),
$$

where $\boldsymbol{\alpha}(\boldsymbol{r})$ is the vector containing the coefficients $\alpha_{j}(\boldsymbol{r})$, for $j \in\left\{1,2, \ldots n_{r}\right\}$. The matrix $\mathcal{U}$ is defined as the matrix $U$ as constructed in the SVD (equation 12), but here it containing only the first $n_{r}$ column vectors. That is, $\mathcal{U}$ is an $n_{m} \times n_{r}$ matrix, and $\mathcal{U}_{i, j}=U_{i, j}$ for $i \in\left\{1,2, \ldots n_{m}\right\}$ and 
$j \in\left\{1,2, \ldots n_{r}\right\}$. This matrix can be used to define a mapping between the POD coefficients and original angular moments (equation 6 ), i.e.

$$
\boldsymbol{\Psi}(\boldsymbol{r})=\mathcal{U} \boldsymbol{\alpha}(\boldsymbol{r}) .
$$

The POD model can now be constructed by applying the Galerkin projection, described in section 2.2 , using the POD basis functions defined in equation 14, and forming the angular discretised equations derived in equation 6. However, rather than applying the Galerkin projection directly, the reduced order model can be formed more efficiently by the discrete projection of the original discretised equations into the POD space. That is, one forms the angular discretised equations 6 in terms of the original spherical harmonic expansion, and then projects onto the POD space by replacing the angular moments with expression 18, and pre-multiplying the system by $\mathcal{U}^{T}$,

$$
\begin{aligned}
\mathcal{U}^{T} A_{x} \mathcal{U} \frac{\partial \boldsymbol{\alpha}}{\partial x}+\mathcal{U}^{T} A_{y} \mathcal{U} \frac{\partial \boldsymbol{\alpha}}{\partial y}+\mathcal{U}^{T} A_{z} \mathcal{U} \frac{\partial \boldsymbol{\alpha}}{\partial z}+\mathcal{U}^{T} H \mathcal{U} \boldsymbol{\alpha} & =\mathcal{U}^{T} S \\
\Rightarrow A_{x}^{P} \frac{\partial \boldsymbol{\alpha}}{\partial x}+A_{y}^{P} \frac{\partial \boldsymbol{\alpha}}{\partial y}+A_{z}^{P} \frac{\partial \boldsymbol{\alpha}}{\partial z}+H^{P} \boldsymbol{\alpha} & =S^{P}
\end{aligned}
$$

This results in a new angular discretised system that is identical in form to the original system in equation 6 . The only difference is that the new angular matrices and vectors formed now relate to the POD discretisation, these operators are all of dimension $n_{r}$ and are denoted with the superscript $P$. The equations' identical structure is attributed to the fact that the SVD was performed on the original snapshot matrix. Had the mean values been removed from the matrix $\mathcal{A}$ prior to the SVD, as in traditional POD methods, additional terms would have formed in equation 20 . These same structures of the angular discretised equations mean that the same spatial treatment (as the full model) can be applied. Boundary conditions can also be treated using the same Riemann method since the approach functions for arbitrary angular discretisations. These properties essentially enable the same code to be used to resolve the POD model in space, in fact only the redefinition of the angular matrices are required. All these properties make the POD model's implementation very efficient and also non-intrusive.

\subsection{Discussion of efficiency}

Whilst computational times for solving the ROM are stated in the following section, this section provides a short discussion on its efficient construction. Firstly, the snapshot matrix constructed from the angular moments is of size $n_{m} \times n_{s}$, where typical angle sizes, $n_{m}$, are within the value of 500 . Assuming the number of finite element nodes, $n_{s}$, will become arbitrarily large, the most efficient way of performing the SVD on $\mathcal{A}$ is to form the $n_{m} \times n_{m}$ matrix $\mathcal{A A}^{t}$. The eigenvalue decomposition of this relatively small symmetric matrix can be found efficiently, and from this the SVD of the matrix $\mathcal{A}$ 
is easily obtainable. The whole process is therefore quite cheap, however there may be an issue of high computational complexity if the number of snapshots become large and computing $\mathcal{A A}^{t}$ expensive. This can be easily overcome by relaxing where the snapshots are taken. It is not necessary to record the snapshots at each spatial node, and instead a smaller selection of points distributed about the problem domain can be used. Provided these points are representative of the flux profiles that form in the problem, the POD model should still function as intended. However the numerical examples presented here use spatial meshes involving only a few thousands finite elements. With this small number of elements the costs of recording the fluxes at all nodes mean the SVD process is efficient; its costs are negligible in comparison to the whole calculation. In addition, once the POD functions have been formed, constructing the POD angular matrices in equation 20 is very efficient. Their constructions are all of order $n_{m}$ or $n_{m}^{2}$, i.e. based on the size of the angular expansion, which is relatively small. Construction of the angular discretised system 20 is negligible in comparison to the full computational costs of its solving.

The POD formulation will involve some additional computational costs (in comparison to other methods) due to the fact that dense angular matrices are formed. Typically any convenient properties of the angular discretisation scheme (such as orthogonality) is lost, and this results in a near 100\% filling of the matrices. In comparison to the $P_{N}$ method this is relatively high since the non-zero filling using this method is of the order 2-3\% (for the larger angular discretisations). As a result, more operations are required in the construction stage using POD, in comparison to $P_{N}$, when the same sized angular expansion are compared, and when the sparseness within the matrices are considered. This does result in an increase in computing times, the implications of which are discussed the following numerical examples.

\section{Numerical Examples}

Two numerical examples are presented in this section to demonstrate the capabilities of the POD model in resolving radiation transport problems. In the first example the propagation of radiation through a 2 dimensional dog-leg duct is solved. This is used to illustrate the capabilities of the model in resolving radiation shielding problems. The second example applies the method to a highly demanding problem involving the emission of radiation from an infinite array of pins. This is based on the pincell model proposed by Adams [37] and is noted for the complex angular flux profiles it generates.

The POD expansion approximations are denoted by $P O D_{N}$, where $N$ has the numeric value equal to the number of POD functions used. These approximations are compared with those of the $P_{N} \mathrm{~s}$; table 
Table 1: Expansion sizes for angular expansion orders. $P_{N}$ sizes are listed for 2 dimensional problems.

\begin{tabular}{ccccccccc}
\hline \hline Expansion size & 3 & 10 & 21 & 36 & 55 & 78 & 120 & 136 \\
\hline$P_{N}$ Order $(N)$ & 1 & 3 & 5 & 7 & 9 & 11 & 13 & 15 \\
$P O D_{N}$ Order $(N)$ & 3 & 10 & 21 & 36 & 55 & 78 & 120 & 136 \\
\hline
\end{tabular}

1 lists the expansion sizes against expansion order $\left(P_{N}\right.$ expansion sizes are listed for 2 dimensional problems).

\subsection{Numerical Example 1: The Dog-Leg Duct}

In this example the transport of radiation through a highly absorbing shield is resolved. The problem domain is presented in figure 2 which shows a source of radiation imbedded within a strongly absorbing material. Adjacent to the source region is a kinked duct containing a low material cross-section that resembles air. This duct allows particles to travel through the shield, by having little interaction with its media, and to escape through its external surface. The duct causes highly directed angular fluxes to form in the problem's solution; preferential directions will form in those angles aimed from the source to the external wall. These directional fluxes are expected to influence the shape of the POD basis functions when trained on these particular geometries. They should become biased in resolving these directions which will, as a result, increase the suitability of the ROM model for solving these problem types. That is, the POD model created from the snapshots of solutions on this geometry should resolve well those problems that form similar flux profiles. This property of the POD model is investigated here.

The POD model was formed from the snapshots of three solutions obtained from the full model. For each of these 'training' problems the shielding material properties were varied, the material crosssections are listed in table 2 - labelled F1-F3. Each solution was obtained using the spatial mesh presented in figure 2, this consisted of 2764 triangular finite elements using 8292 (discontinuous FE) spatial nodes, together with a high resolution $P_{21}$ angular approximation. With this discretisation there were a total of 24876 snapshots, each being vectors of size 253 . The POD model constructed from this snapshot data was then tested by using it to resolve 8 shielding problems. Three of these problems were identical to the training problems; however the other five were different by the varying of the material properties of the shield. The material cross-sections for each problem are also listed in table 2 and are labelled R1-R8. The geometry and mesh were kept the same for all training and testing calculations. 

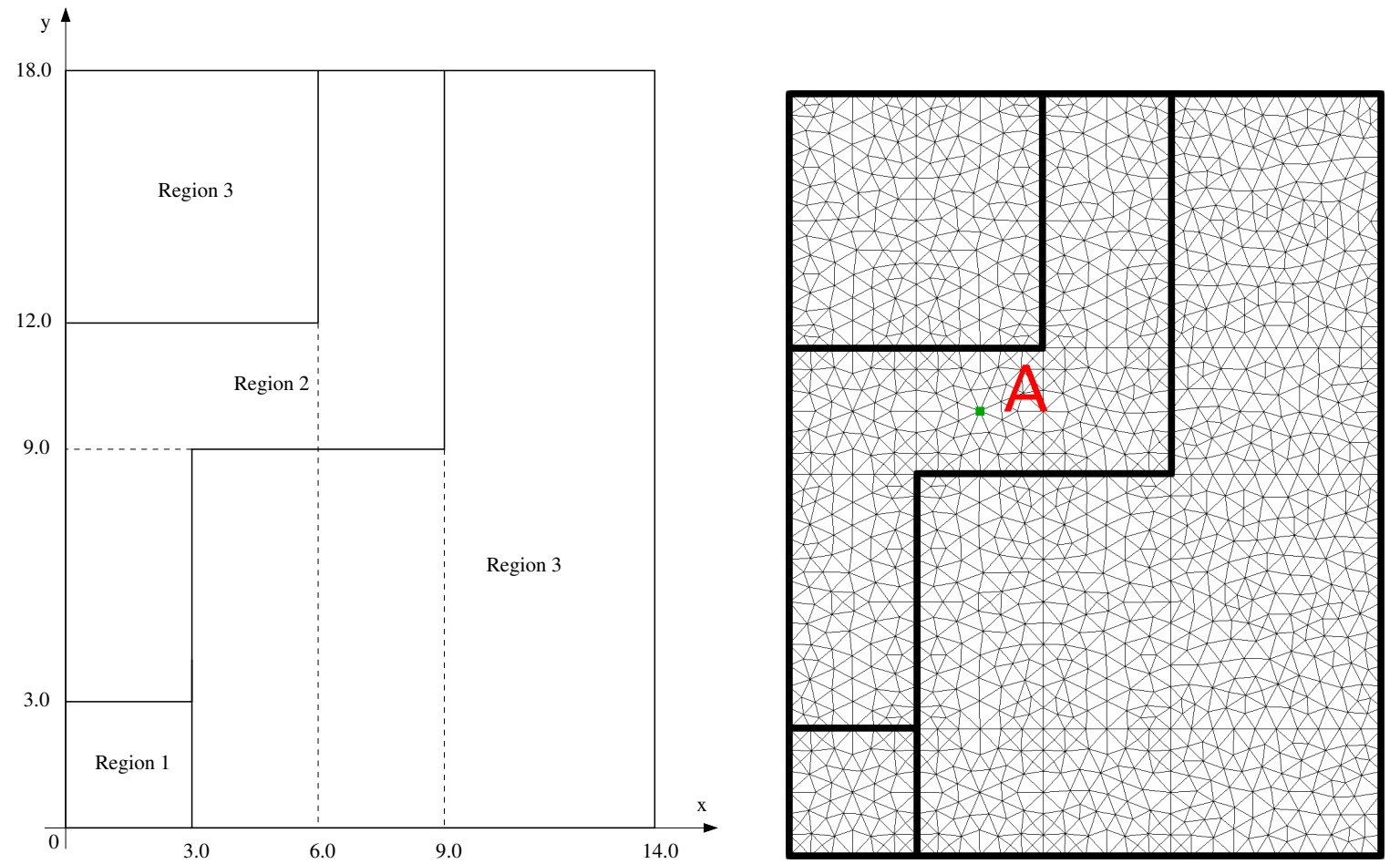

Figure 2: The domain and spatial mesh for numerical example 1.
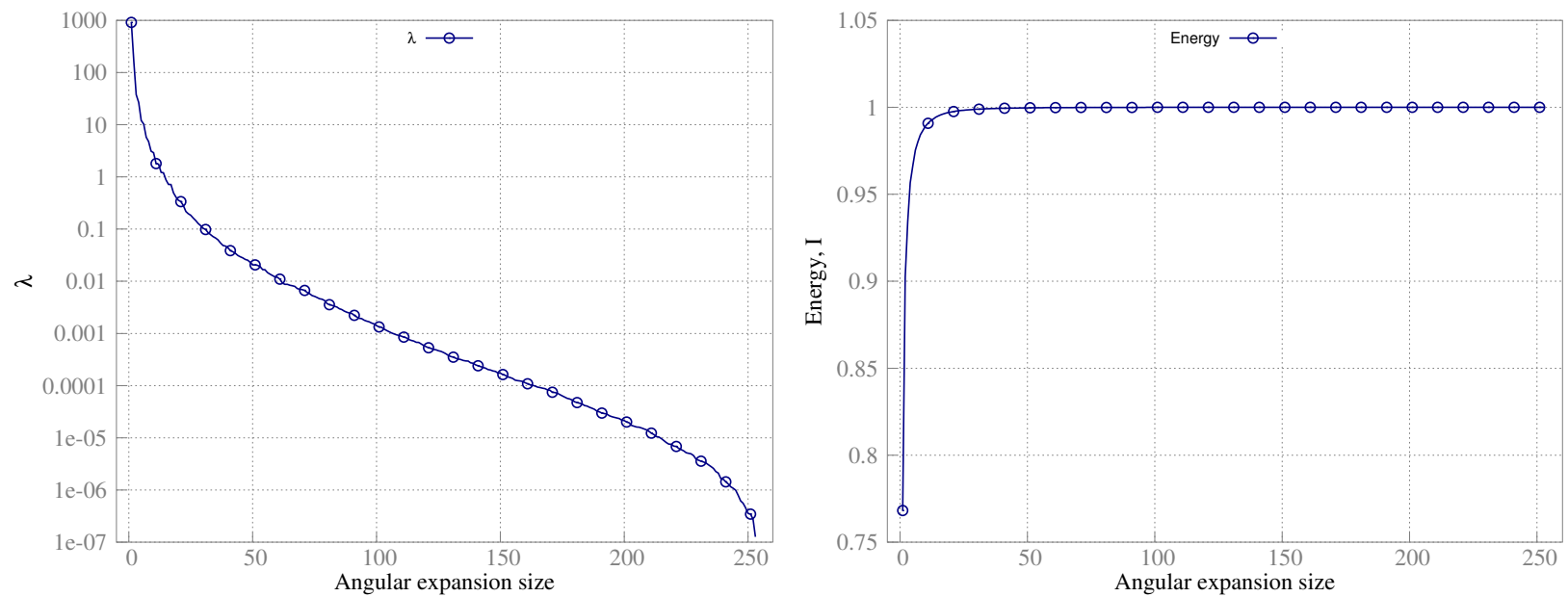

Figure 3: SVD analysis of the snapshot matrix in the dog-leg duct problem. Left: the squared singular values $\lambda_{i}=\sigma_{i}^{2}$, Right: the energy $I$ captured as a function or retained POD functions. 
Table 2: Material cross-section specifications for the dog-leg duct problems.

\begin{tabular}{|c|c|c|c|c|}
\hline Test Case & Cross-sections & Region 1 & Region 2 & Region 3 \\
\hline \multirow[t]{2}{*}{ F1/R1 } & $\Sigma_{a} / \mathrm{cm}^{-1}$ & 1.00 & 0.01 & 5.00 \\
\hline & $\Sigma_{s} / \mathrm{cm}^{-1}$ & 0.00 & 0.00 & 0.00 \\
\hline \multirow[t]{2}{*}{$\mathrm{F} 2 / \mathrm{R} 2$} & $\Sigma_{a} / \mathrm{cm}^{-1}$ & 1.00 & 0.01 & 3.00 \\
\hline & $\Sigma_{s} / \mathrm{cm}^{-1}$ & 0.00 & 0.00 & 2.00 \\
\hline \multirow[t]{2}{*}{ F3/R3 } & $\Sigma_{a} / \mathrm{cm}^{-1}$ & 1.00 & 0.01 & 1.00 \\
\hline & $\Sigma_{s} / \mathrm{cm}^{-1}$ & 0.00 & 0.00 & 4.00 \\
\hline \multirow[t]{2}{*}{$\mathrm{R} 4$} & $\Sigma_{a} / \mathrm{cm}^{-1}$ & 1.00 & 0.01 & 4.00 \\
\hline & $\Sigma_{s} / \mathrm{cm}^{-1}$ & 0.00 & 0.00 & 1.00 \\
\hline \multirow[t]{2}{*}{$\mathrm{R} 5$} & $\Sigma_{a} / \mathrm{cm}^{-1}$ & 1.00 & 0.01 & 2.00 \\
\hline & $\Sigma_{s} / \mathrm{cm}^{-1}$ & 0.00 & 0.00 & 3.00 \\
\hline \multirow[t]{2}{*}{$\mathrm{R} 6$} & $\Sigma_{a} / \mathrm{cm}^{-1}$ & 1.00 & 0.01 & 3.00 \\
\hline & $\Sigma_{s} / \mathrm{cm}^{-1}$ & 0.00 & 0.00 & 3.00 \\
\hline \multirow[t]{2}{*}{$\mathrm{R} 7$} & $\Sigma_{a} / \mathrm{cm}^{-1}$ & 1.00 & 0.01 & 4.00 \\
\hline & $\Sigma_{s} / \mathrm{cm}^{-1}$ & 0.00 & 0.00 & 2.00 \\
\hline \multirow[t]{2}{*}{$\mathrm{R} 8$} & $\Sigma_{a} / \mathrm{cm}^{-1}$ & 1.00 & 0.01 & 3.50 \\
\hline & $\Sigma_{s} / \mathrm{cm}^{-1}$ & 0.00 & 0.00 & 3.50 \\
\hline
\end{tabular}


The singular values resulting from the SVD of the snapshot data are presented in the graph of figure 3. This figure also presents the graph showing the snapshot energy retained as a function of the number of POD basis functions - as given in equation 15. It is shown that there is a drop of nearly three orders of magnitude in the squared value of the 10th singular value in comparison to the first. The singular values continue to drop off at a steady rate indicating that the POD basis functions are capturing the energy of the data very efficiently. This is confirmed on inspection of the energy graph which shows that 21 POD basis functions retain $99.7 \%$ of the information of the snapshots.

The first eight POD basis functions are presented in figure 4. These clearly show that there is preferential direction given those the angles in line with the directions propagating particles through the duct. This biasing means that the resolution is being placed in the important regions of angle that are required to resolve the problem accurately. Figure 5 provides some evidence for this by comparing the scalar flux solutions from the high resolution (exact) $P_{21}$ approximation with those of a low resolution $P_{3}$ and $P O D_{10}$ approximations (both of which use 10 basis functions). These solutions were obtained from solving the unseen problem R5. The profiles clearly show that the POD solutions are in very close agreement with the exact solution. They also show that the low order $P_{N}$ method has struggled to resolve the duct region - its solution through the duct is highly diffuse. Figure 6 presents the graphs showing the scalar flux profiles across the problem's boundary at the mouth of the duct. Each graph compares the exact flux with the solutions obtained from POD and $P_{N}$ using the same number of basis functions. It is evident that the POD approximation provides substantially improved approximations in comparison to the $P_{N}$ method, when using the same number of basis functions. In particular, for the low order approximations involving 21 or less basis functions, the POD model captures the general magnitude of the duct well, whilst the $P_{N}$ method overestimates its value by several factors. For the higher resolution expansions the $P_{N}$ method is still some way off from resolving the duct accurately, whilst the POD method has captured the flux's detail very well.

Figures 7 and 8 present the angular flux profiles of the exact, $P_{N}$ and POD solutions at the location in the duct indicated in figure 2 (again when solving problem R5). The exact profiles presented figures 7 are in fact showing the same solutions; one shows the flux as a function on the sphere's surface, whilst the other also projects the surface, according to the flux's magnitude (this article varies between these visualisations in order to help see the flux detail). These profiles compare the solutions of POD and $P_{N}$ using 10, 21 and 55 basis functions respectively. Whilst there are clear differences in the low order solutions, in comparison to the exact solution, the POD solution has still managed to form the dominant peak in the flux. The low order $P_{N}$ solution on the other hand has not formed the peak well, it is shown to be smeared across large parts of the angular domain. The fluxes approximated using 21 basis functions show the POD model to be in close agreement with the reference solution, whilst the $P_{N}$ has still yet to resolve the peak. It is only when the larger expansions are used that the $P_{N}$ approximation begins to form the peak, although there are still visible differences. However at 

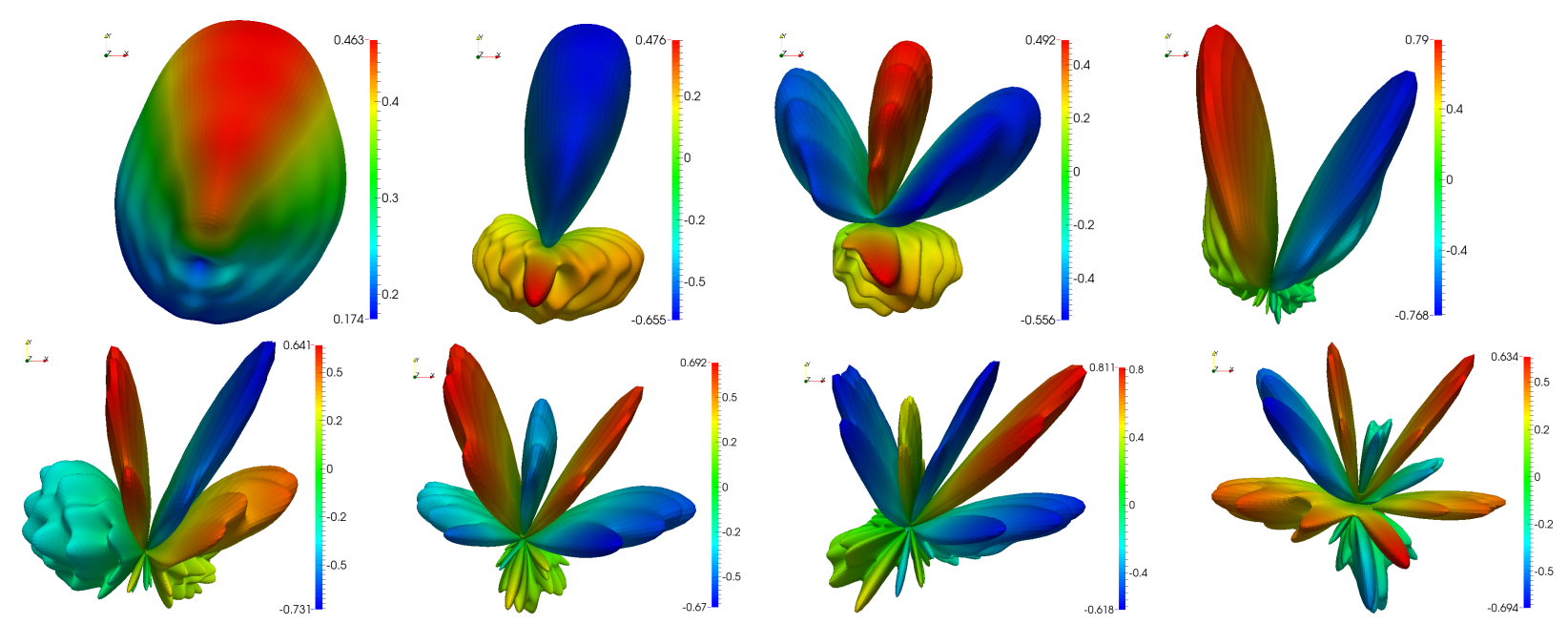

Figure 4: The first 8 POD basis functions formed in the dog-leg duct problem - all are viewed facing in the x-y plane, i.e. in line with the diagram of figure 2 .
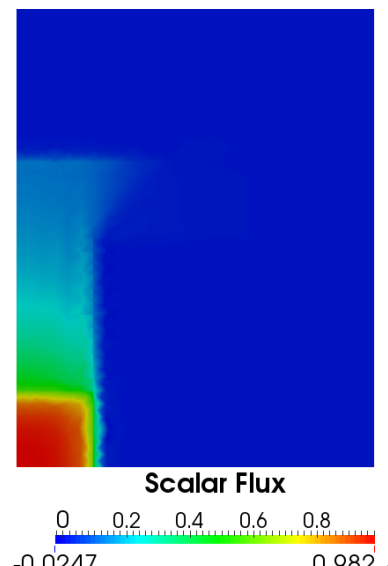

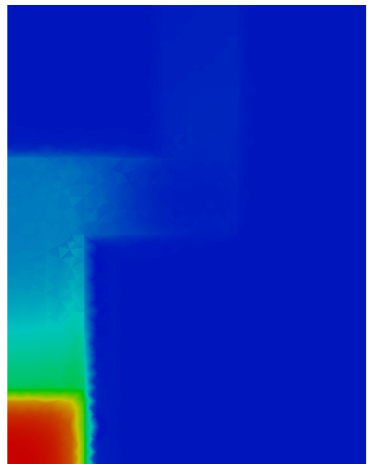

Scalar Flux

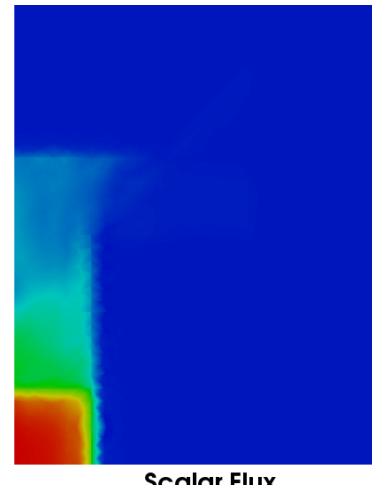

Scalar Flux

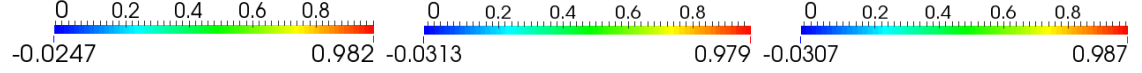

Figure 5: Scalar flux solution for problem R5. Left to Right: $P_{21}$ (Exact), $P_{3}$ (10 Basis functions), $P O D_{10}(10$ basis functions). 

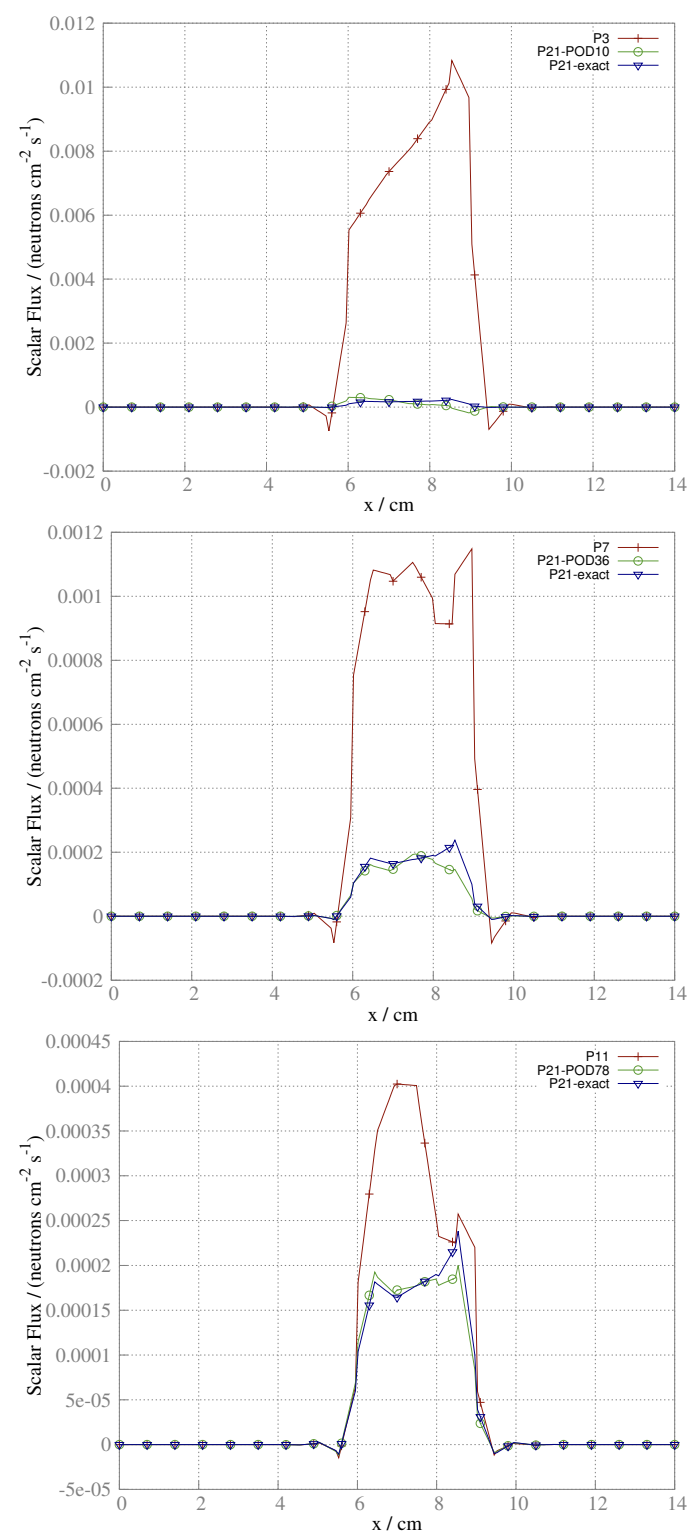
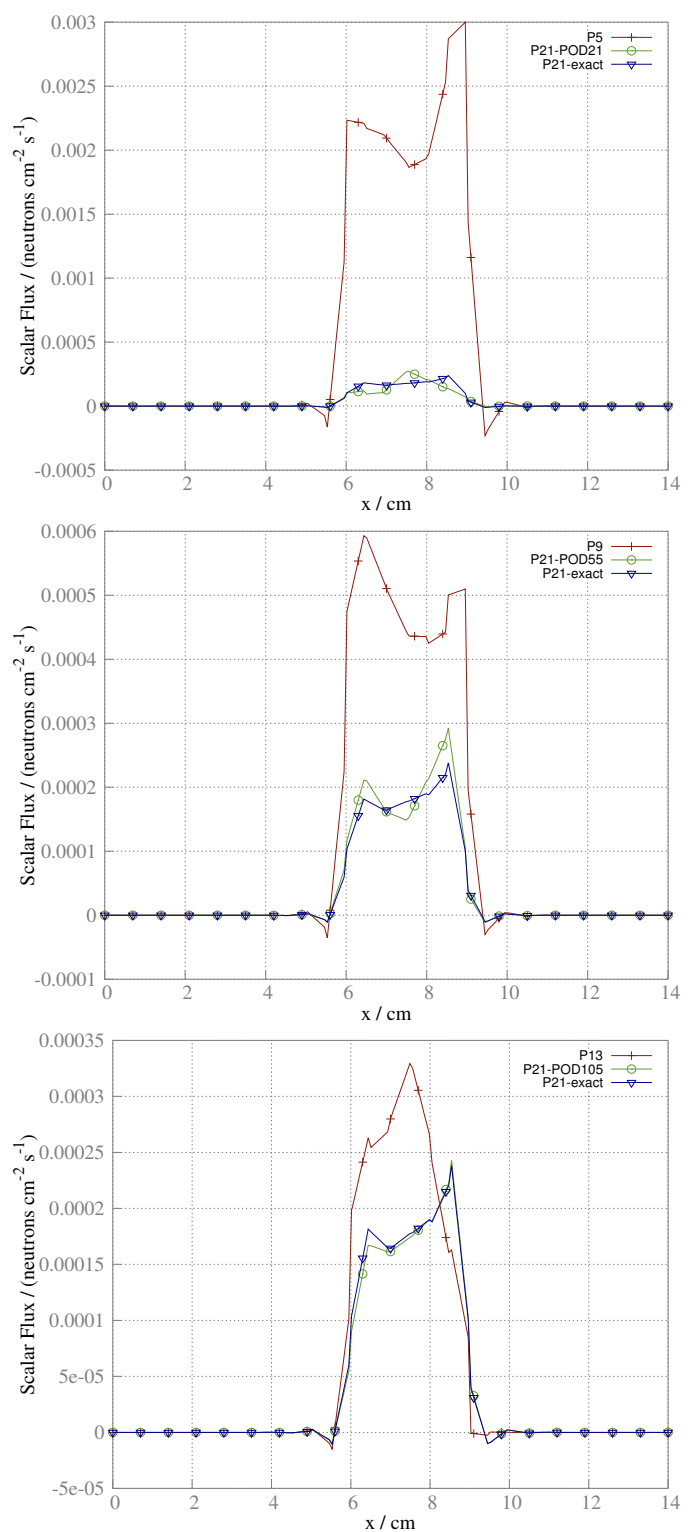

Figure 6: Scalar flux profiles along the top boundary $((0.0,18.0)$ to $(14.0,18.0))$ of the dog-leg duct problem R5. Leftright, top-bottom: Comparisons of the POD and $P_{N}$ solutions using 10, 21, 36, 55, 78, 105 angular basis functions. 


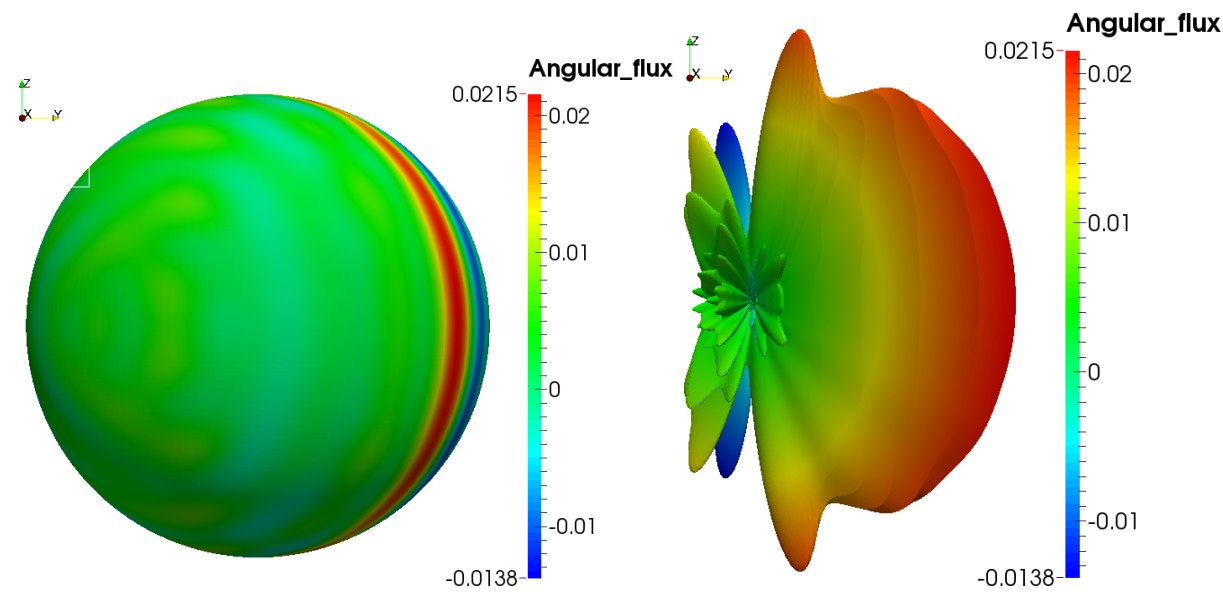

Figure 7: The exact angular flux profiles at position A of the dog-leg duct problem - material specification R5. Left: Shown as a function on the sphere. Right: Shown as a projected function.

this point the POD model using the same number of basis functions has reconstructed the flux very well - there are few visible differences with the reference solution.

The graphs in figures 9 and 10 present the scalar and angular flux error norms for problems R3-R8. These error metrics for angular (at some position $\boldsymbol{r}$ ) and scalar fluxes are defined as,

$$
E_{a}=\int_{\Omega}\left(\psi_{\text {app }}(\boldsymbol{r})-\psi_{\text {exact }}(\boldsymbol{r})\right)^{2} d \Omega
$$

and

$$
E_{s}=\int_{V}\left(\phi_{\text {app }}-\phi_{\text {exact }}\right)^{2} d V
$$

respectively. The term $\phi=\int_{\Omega} \psi d \Omega$ denotes the scalar flux, and the subscripts app and exact refer the fluxes as being approximate and exact.

The error graphs show the POD model to have stronger rates of convergence in comparison to the $P_{N}$ model. It is also notable that the low order POD models generate solutions that are as accurate as high order $P_{N}$ solutions. For example, many of the graphs show that the $P O D_{10}$ solution has roughly the same accuracy as $P_{15}$. The sizes of these angular expansions are 10 and 136, respectively, showing a significant reduction in problem size. The POD model has also performed well on all problems including those not used in training. This supports this paper's view that the method should work effectively on problems that generate similar angular flux profiles to those used during testing. 


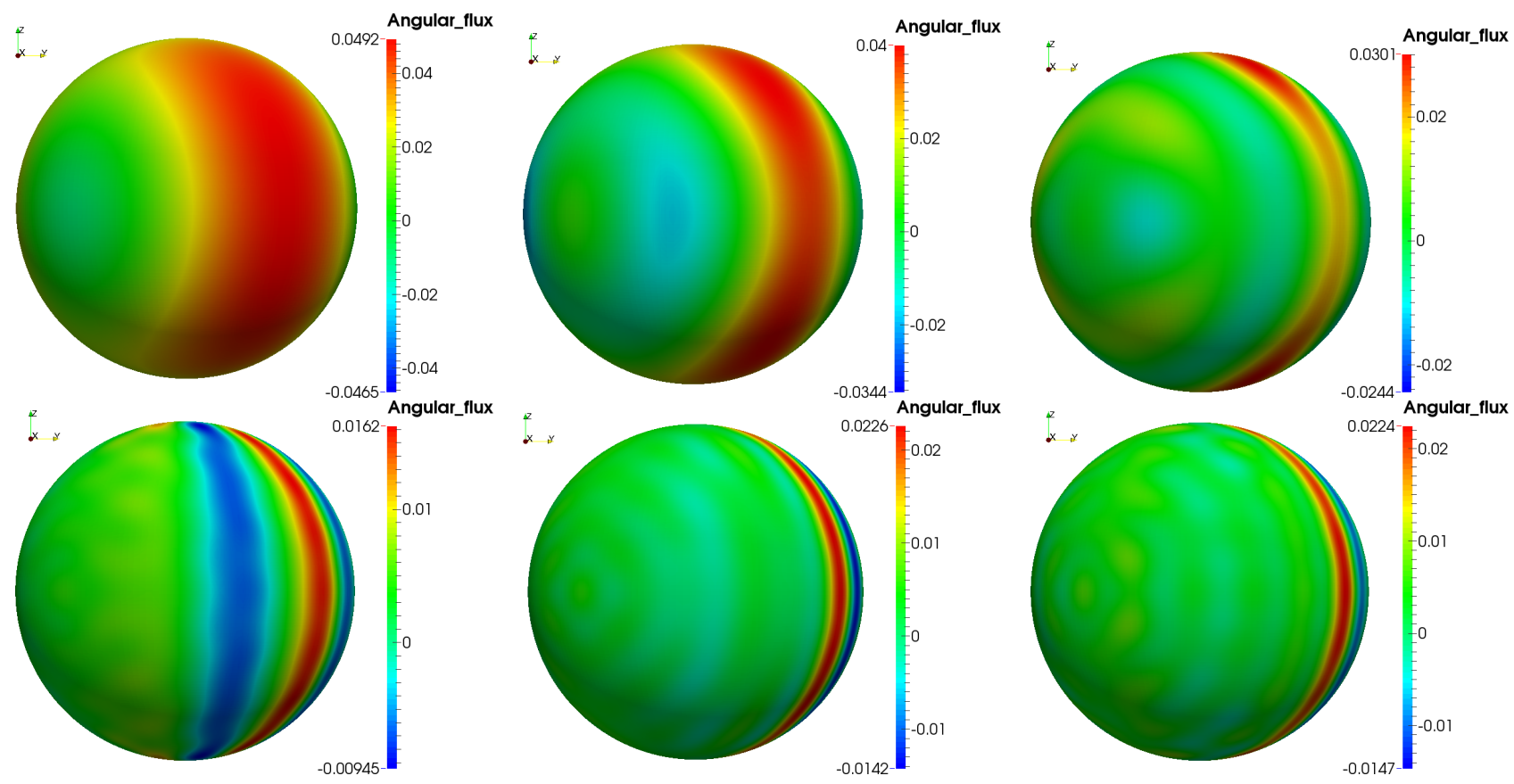

Figure 8: Angular flux profiles at position A of the dog-leg duct problem - material specification R5. Top: $P_{3}, P_{5}, P_{9}$ solutions, Bottom: $P O D_{10}, P O D_{21}, P O D_{55}$ solutions. 
Table 3 presents the computational times and memory costs required to generate and solve the POD and $P_{N}$ models for the unseen problem R5. The figures compare the computing times to solve the models when using the same angular expansion size; the results show them to require roughly equal times (for comparable sized expansions). However the breakdown of these times show that for the solving stage, the POD model requires approximately half the time as that of the $P_{N}$ model. This is an interesting outcome since identical solvers were used to solve each model (a PETSC GMRES solver [38] with SSOR preconditioning), which meant that each solver iteration would have required approximately the same computing effort. To get this reduction in time the POD model would have therefore required around half the iterations as that of the $P_{N}$ model. This reduction in solver iterations hints towards there being more stable linear systems formed using POD. The computing times also mean that the construction of the POD model requires more time to construct in comparison to the $P_{N}$ model. This is due to the fact that all sparseness in the angular matrices is lost when constructing the POD model, as already discussed in section 2.5. The memory requirements stated in the table show the number of floating point numbers per FE nodal connection needed to store the matrix. For a given angular size both the $P_{N}$ and POD models require the same storage space, which increases as a quadratic function of expansion size. This rapid rise in storage requirements (as angle sizes increase) demonstrate a further benefit of the POD model, since significantly smaller expansion sizes, requiring less memory, can be used without compromising accuracy.

Figure 11 presents these computational times plotted against their scalar flux error norms (the errors are calculated from the solution obtained from the respective angular expansion). This result shows that the POD model significantly increases computational efficiency. For higher orders of accuracy, the reduction in computational time approaches 2 orders of magnitude. An indication from the graphs' gradients is that the efficiency will increase further with the higher accuracy being sought.

\subsection{Numerical Example 2: Adams Pincell}

This section resolves the Adams Pincell problem [37] which involves the transport of radiation emitted from an infinite array of pins. The problem domain is presented in figure 12 which shows a square source region enclosed within a box that has reflective boundary conditions applied to all sides. The material properties of the problem are listed in table 4 .

The problem was spatially resolved using the mesh presented in figure 12 which consisted of 3602 triangular finite elements and 10806 (discontinuous FE) spatial nodes. The angular direction was resolved using a high resolution $P_{21}$ discretisation. Figure 13 presents the resulting scalar flux approximation which, in this article, is assumed to be the exact solution. On first inspection, the pincell solution looks 

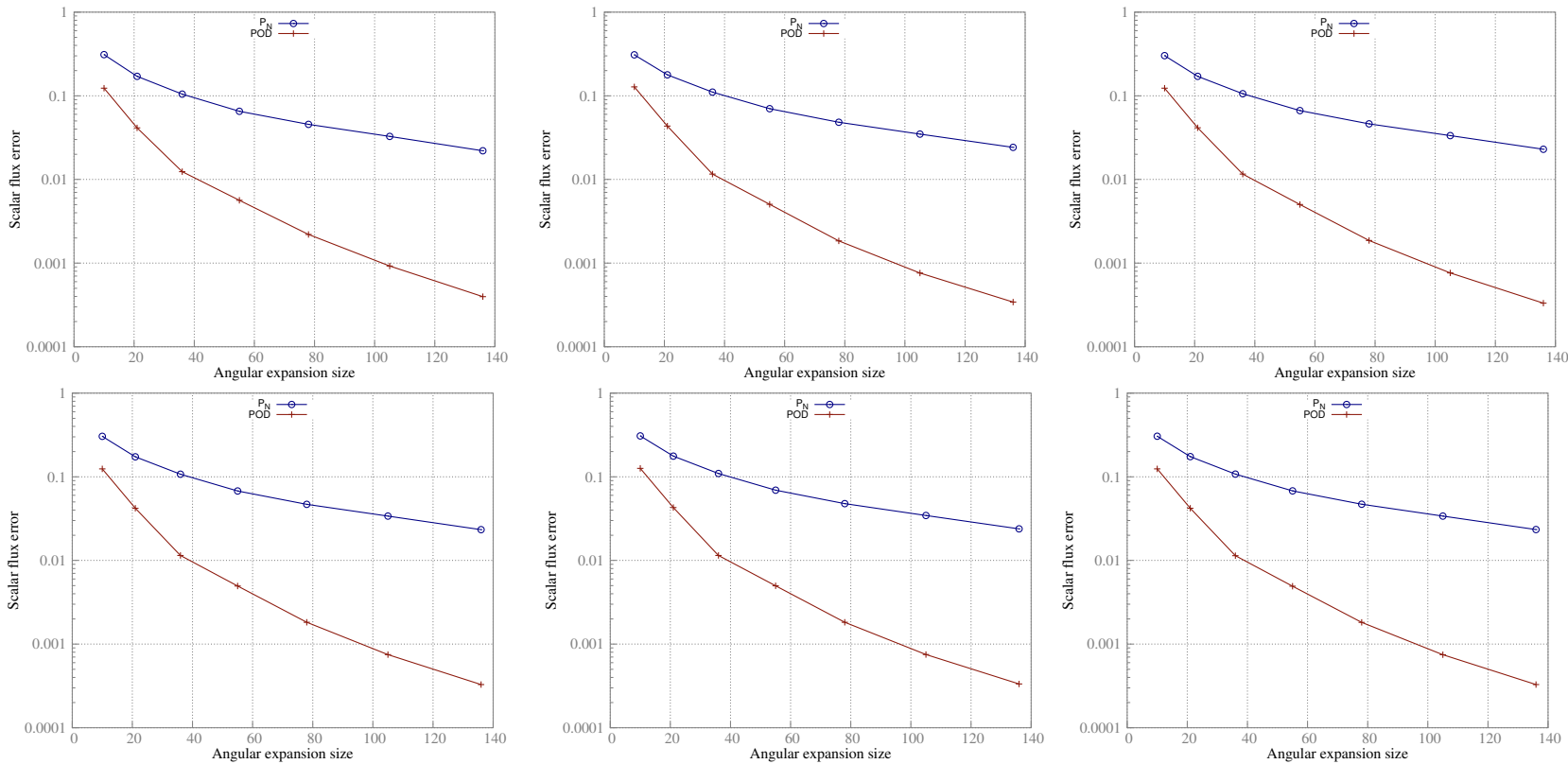

Figure 9: Scalar flux error norms for the $P_{N}$ and $P O D$ approximations for problems R3 to R8 (left to right, top to bottom).
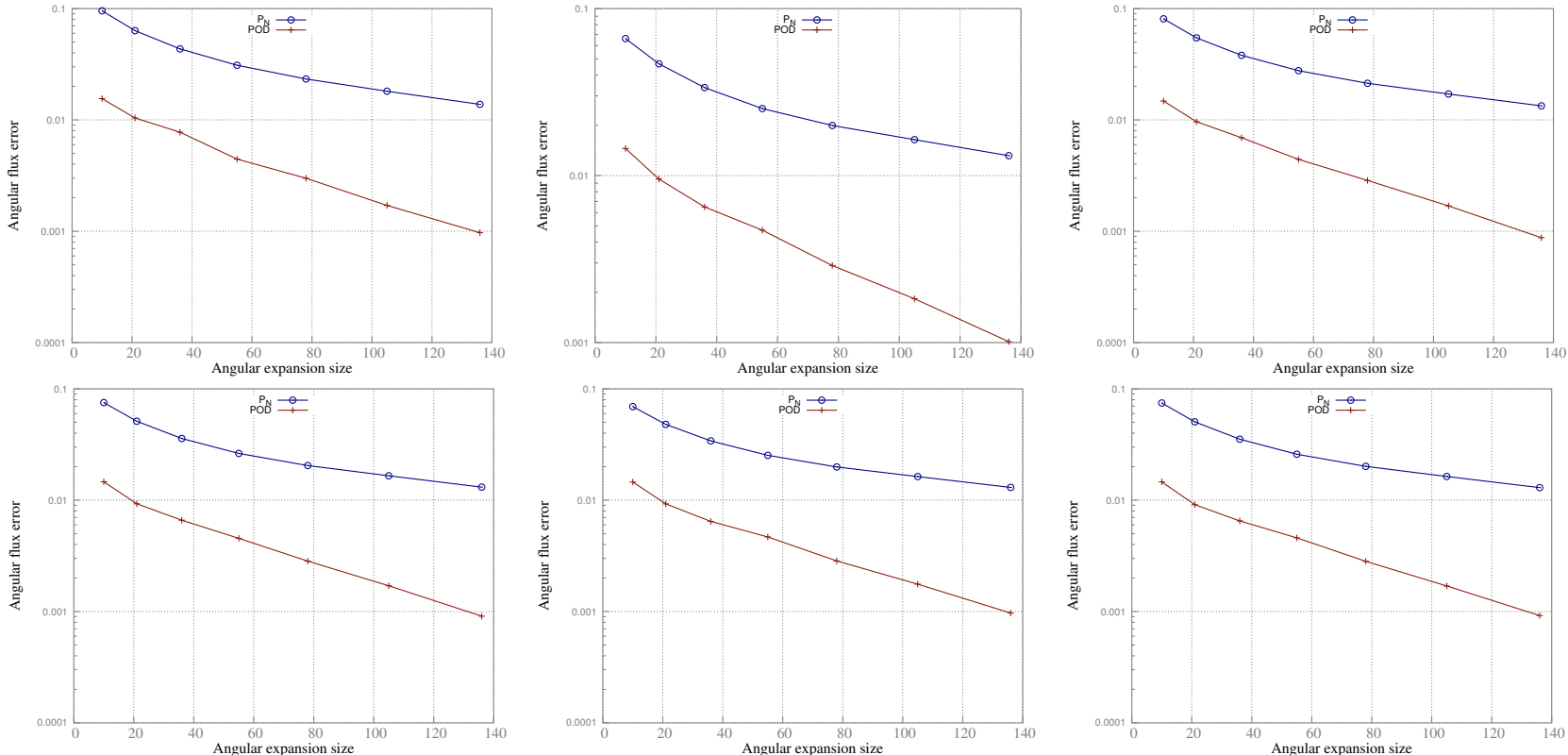

Figure 10: Angular flux error norms for the $P_{N}$ and $P O D$ approximations for problems R3 to R8 (left to right, top to bottom) at position $\mathrm{A}$. 
Table 3: Computational solving times and storage requirements (floating point numbers per FE node connection) for the dog-leg duct problem using material specification R5. Overall time include all setup and solving times.

\begin{tabular}{l|ccc}
\hline \hline & Overall time / s & Solver time / s & Memory costs \\
\hline$P 21$-exact & 3117.0 & 1003.2 & 64009 \\
\hline$P 3$ & 1.6 & 0.5 & 100 \\
$P 21-P O D 10$ & 1.1 & 0.2 & \\
\hline$P 5$ & 6.8 & 3.7 & 441 \\
$P 21-P O D 21$ & 4.6 & 1.5 & \\
\hline$P 7$ & 22.8 & 12.3 & 1296 \\
$P 21-P O D 36$ & 21.3 & 9.5 & \\
\hline$P 9$ & 61.2 & 31.2 & 3025 \\
$P 21-P O D 55$ & 55.1 & 19.7 & \\
\hline$P 11$ & 144.2 & 67.2 & \\
$P 21-P O D 78$ & 136.5 & 42.3 & 11025 \\
\hline$P 13$ & 350.6 & 177.0 & 18496 \\
$P 21-P O D 105$ & 304.3 & 89.5 & \\
\hline$P 15$ & 662.4 & 306.1 & \\
$P 21-P O D 136$ & 612.7 & 168.9 & \\
\hline \hline
\end{tabular}

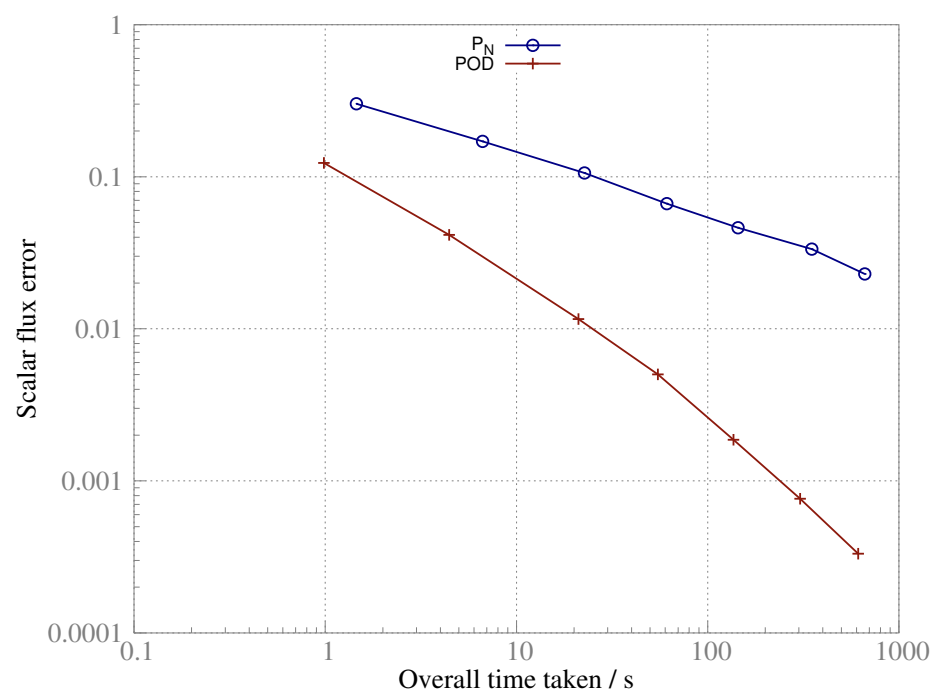

Figure 11: Modelling accuracy for the $P_{N}$ and $P O D$ approximations as a function of computational time to solve. 
rather modest in complexity; its profile is diffuse and smoothly varying. However it is on inspection of the angular flux profiles that the problem's complexity is highlighted. An example is presented in figure 14 which shows the profile of the angular flux at the spatial positions indicated in figure 12. It shows the variation in the angular flux through the azimuthal angle $\omega$ with the polar angle fixed at $\mu=0.33$. The angular flux has sharp oscillations which is a result of the infinite arrangement of the pins causing peaks and shadows to form when the angles align with the other pins. It is these types of profiles that present difficulties for the angular discretisation to construct.

The single $P_{21}$ solution presented in figure 13 was used to form the snapshot matrix, which is of size $253 \times 10806$, from which the POD model was formed. The singular values resulting from the SVD of the snapshot matrix are presented in figure 15. For this example the reduction in the singular values is extremely fast, the tenth squared singular value is almost five orders of magnitude smaller than the first. Figure 15 also presents the retention of snapshot information as a function of the retained POD functions - see equation 15 . One basis function alone captures above $99.2 \%$ of the information whilst ten basis functions exceed $99.9 \%$.

The first 6 POD basis functions are presented in figure 16. They are shown to have larger variation through the azimuthal angle as opposed to the polar angle. This corresponds to the flux profiles that would be expected to form within a $2 \mathrm{D}$ array of pins, aligned in the $\mathrm{x}-\mathrm{y}$ plane, as greater flux variations would occur in these directions. The pincell problem was reconstructed using the POD model and figure 13 compares the $P O D_{10}$ scalar flux solution with that of $P_{3}$. In comparison to the exact solution there appears to be only minor differences between all three solutions. However the solution profiles plotted across the diagonal of the domain, which are presented in figure 17 , show that greater accuracy is obtained when using POD. In fact the results show that even the low order $P O D_{10}$ discretisation captures the solution's profile and peak very well. Note that not even the higher order $P_{13}$ approximation (using 105 basis functions) was able to capture the profile as accurately. For the higher order POD approximations, there are no visible differences in comparison to the exact solution.

The $L^{2}$ error norms for the scalar flux solutions are plotted in figure 18. It reveals POD to have performed well and that $P O D_{10}$ produces smaller errors than $P_{15}$. Moreover, the difference in the error norms start from one order of magnitude but this difference increases very quickly - the order of convergence is significantly faster for POD than $P_{N}$. 
Table 4: Material cross-section specifications for the Adam's pin cell problem.

\begin{tabular}{cccc}
\hline \hline Test Case & Cross-sections & Region 1 & Region 2 \\
\hline F1/R1 & $\Sigma_{a} / \mathrm{cm}^{-1}$ & 0.083524 & 0.064132 \\
& $\Sigma_{s} / \mathrm{cm}^{-1}$ & 0.057843 & 0.008642 \\
\hline \hline
\end{tabular}
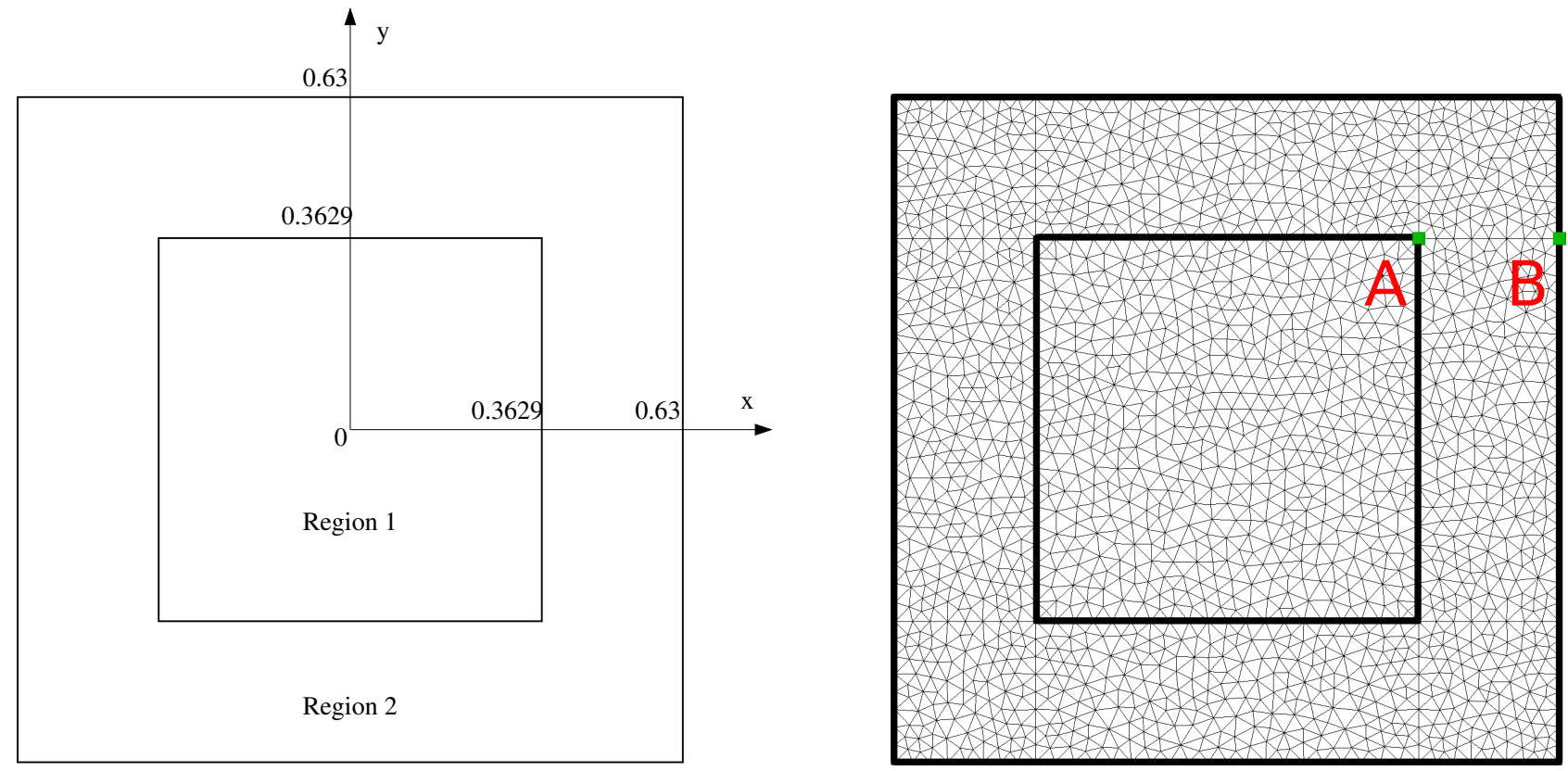

Figure 12: The domain and spatial mesh of the Adam's pin cell problem.


Figure 13: Scalar flux solution profiles of the Adam's pincell problem. Left to right: Exact $\left(P_{21}\right), P_{3}$ and $P O D_{10}$. 


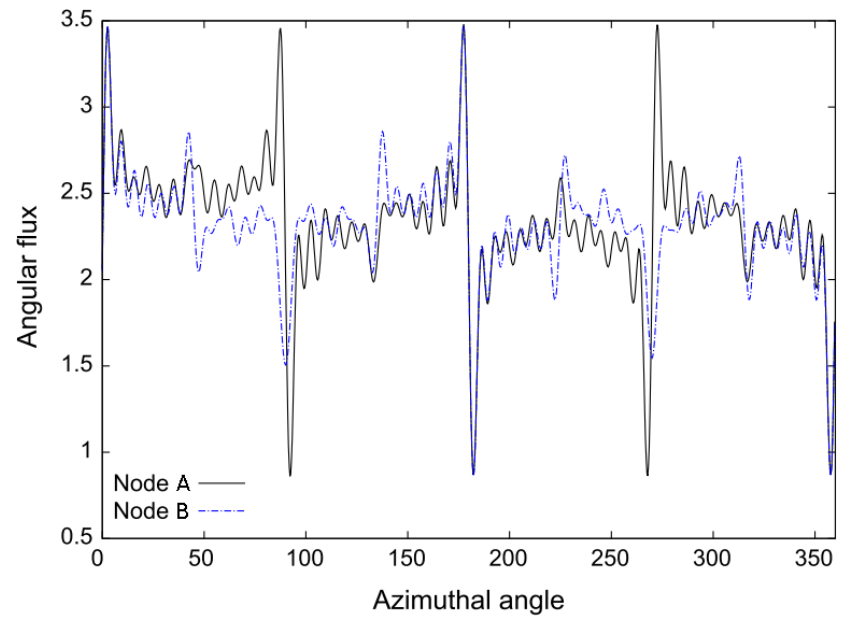

Figure 14: Angular flux at positions A \& B through the azimuthal direction $\omega \in[0,360]$ (in degrees) at polar direction $\mu=0.33$ - taken from [39].
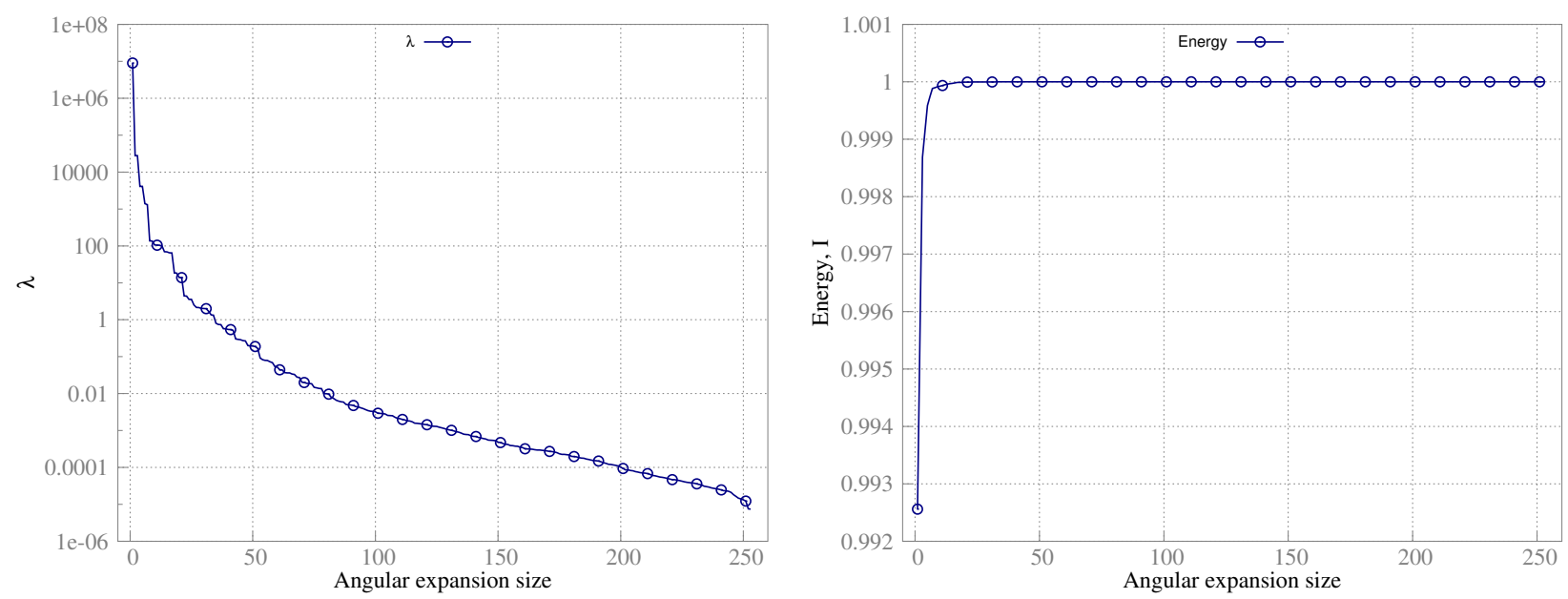

Figure 15: SVD analysis of the snapshot matrix in the Adam's pincell problem. Left: the squared singular values $\lambda_{i}=\sigma_{i}^{2}$, Right: the energy $I$ captured as a function or retained POD functions. 


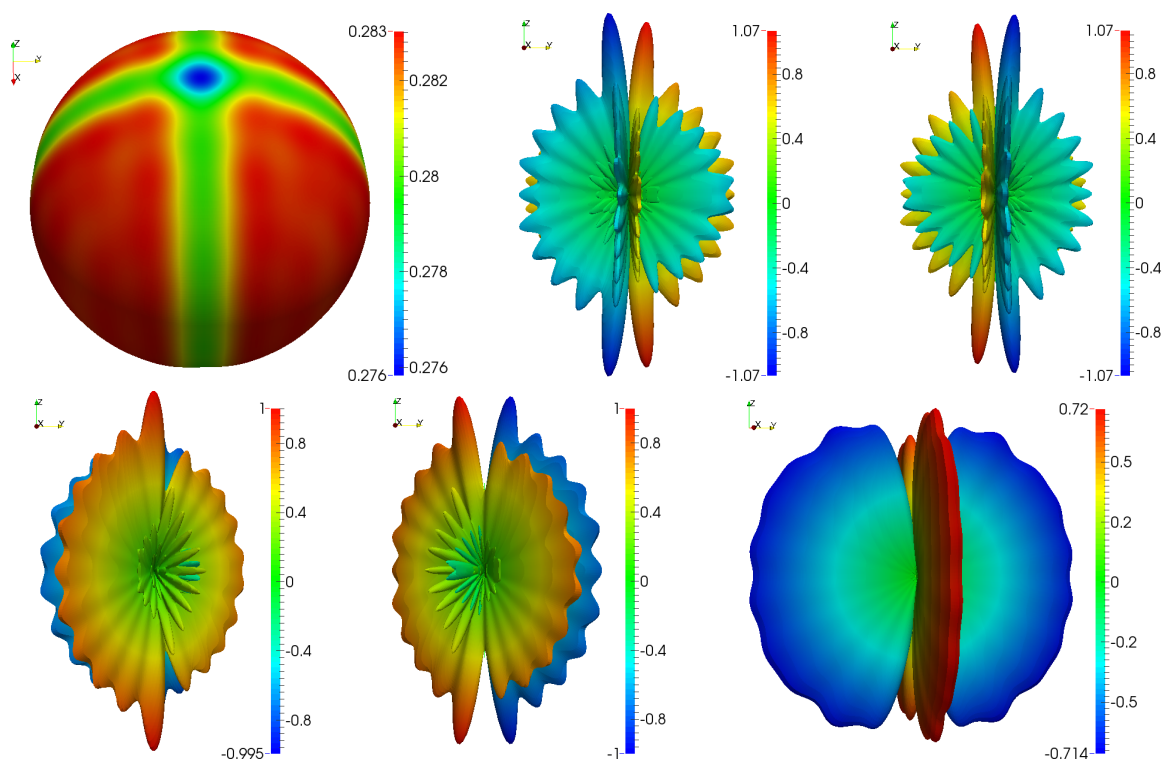

Figure 16: The first 6 POD basis functions formed in the Adam's pincell problem. 
The angular flux profiles at point $\mathrm{A}$, as indicated in figure 12 , for the exact, POD and $P_{N}$ approximations are presented in figure 19. The oscillatory behaviour in the exact flux profiles is shown to be captured well by all POD solutions of varying resolution. However the slow convergence of the $P_{N}$ method is highlighted in these results, since even the largest of approximations is far from capturing all the detail. Figures 20 and 21 present the flux through the azimuthal angle $\omega$, for the polar angle $\mu=0.33$, at the positions 1 and 2 as indicated in figure 12. Again these highlight how well POD model performs. The low order POD approximations, using just 10 basis functions, manage to capture the general shape of the flux oscillations, whilst the high order approximations are in close agreement with the exact solution. The graphs show that the $P_{N}$ method requires the high order $P_{15}$ approximation in order to capture the general oscillations, however there are still clear differences with the exact solution.

The $L^{2}$ error norms for position 2 are plotted in the graph of figure 22. This result shows that as the resolution is increased the POD model converges at a much faster rate. The POD model also appears to perform well when using low order expansion, which is in agreement with the results already presented. Errors from the $P O D_{10}$ expansion are shown to be smaller than those using $P 15$.

\section{Conclusion}

This article has presented a new POD based reduced order model for discretising the angular dimension of the Boltzmann transport equation. It forms optimal angular basis functions for resolving particles' direction of travel by applying an SVD to the snapshot matrix formed from the solutions of a high resolution model. A unique aspect of this method is that the snapshot data is formed from the solution vectors containing angular coefficients. In addition these vectors are recorded through space, as opposed to time, and it is this approach that allows for time independent problems to be solved (which is similar to that of $[23,24]$ ). It has been shown that the angular discretised system of equations derived from the POD formulation can be constructed very efficiently. This reduced order angular discretised system also retains the same structure as that formed by the full model. This allows for the same finite element formulation and Riemann method to be used in resolving the spatial dependence and boundary conditions, respectively. This re-use of methods made the implementation of the POD model particularly simple, elegant and effectively non-intrusive.

The numerical examples presented in this article were chosen to demonstrate the capabilities of the POD model as they formed particularly demanding angular flux profiles to capture. In the first example a shielding application was solved, where the POD model was trained on a small set of 

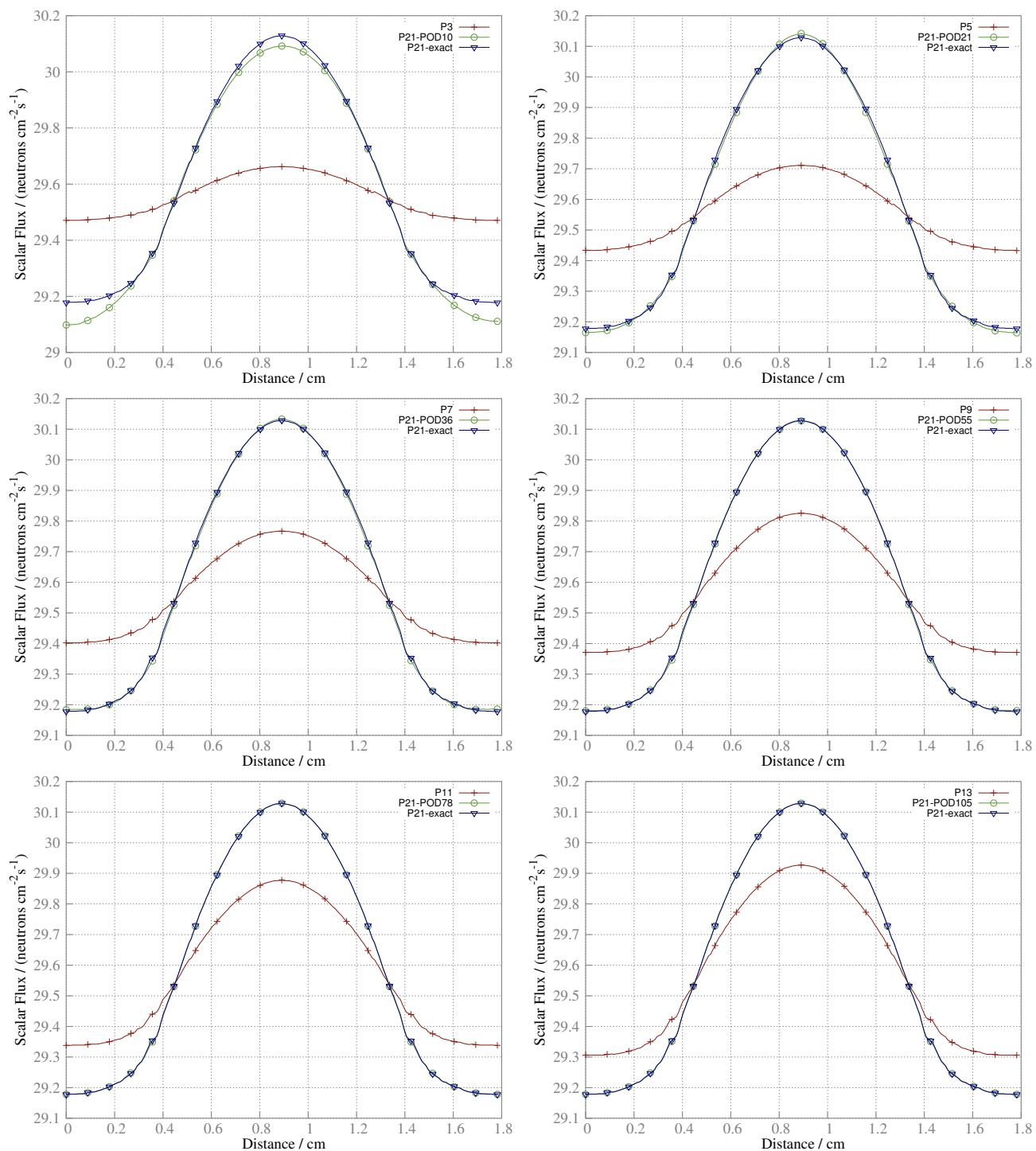

Figure 17: Scalar flux solution profiles through the problem's diagonal $((-0.63,-0.63)-(0.63,0.63))$ using the $P 3$, $P O D_{10}$ approximations. 


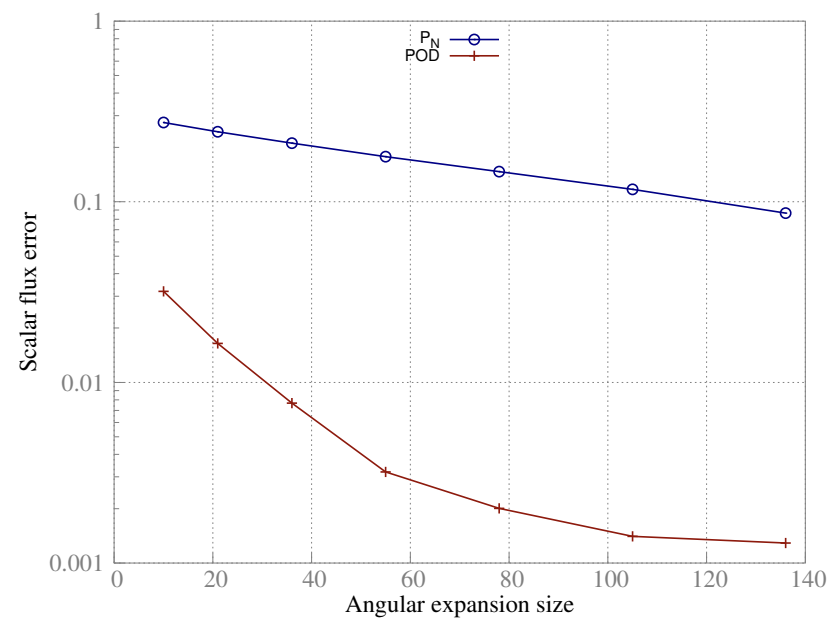

Figure 18: Scalar flux error norms for increasing $P_{N}$ and POD resolution.

problems and then tested on un-seen cases where the material properties were varied. It was shown that for these examples where similar flux profiles would form, the POD model was extremely accurate (even for those problems not used during training). In comparison to the $P_{N}$ method the size of the problem could be reduced by two orders of magnitude and still retain the same accuracy; computational times could be reduced by a similar amount. However as the order of convergence was shown to be significantly better for the POD model, these efficiency gains could be improved further still.

For the second numerical example the POD model was shown to accurately resolve the pincell's solution whilst maintaining a high degree of efficiency. Although the POD model was used to reconstruct the same problem used during its training, this result still provides evidence that the model is suitable for resolving similar unseen problem types. The POD model could therefore greatly benefit reactor physics problems that involve thousands of fuel pins with varying material properties but similar flux profiles. The potential to reduce these problems sizes by two orders of magnitude or more would bring huge benefits to these problems. This will be the focus of future work.

The numerical examples presented in this article have also demonstrated that the memory requirements, when using POD, can be drastically reduced when the matrix to be solved is stored. In fact, if the angular reduction using POD is sufficient enough, the possibility of storing the matrix for large scale problems becomes real. Stored matrix methods are currently not employed in most industry standard models due to the excessive memory requirements. As a direct result of this, the current underlying discretisation methods are restricted to matrix-free SN sweep based methods such as those discussed in [40]. This POD approach therefore offers a potential alternative to such schemes. 


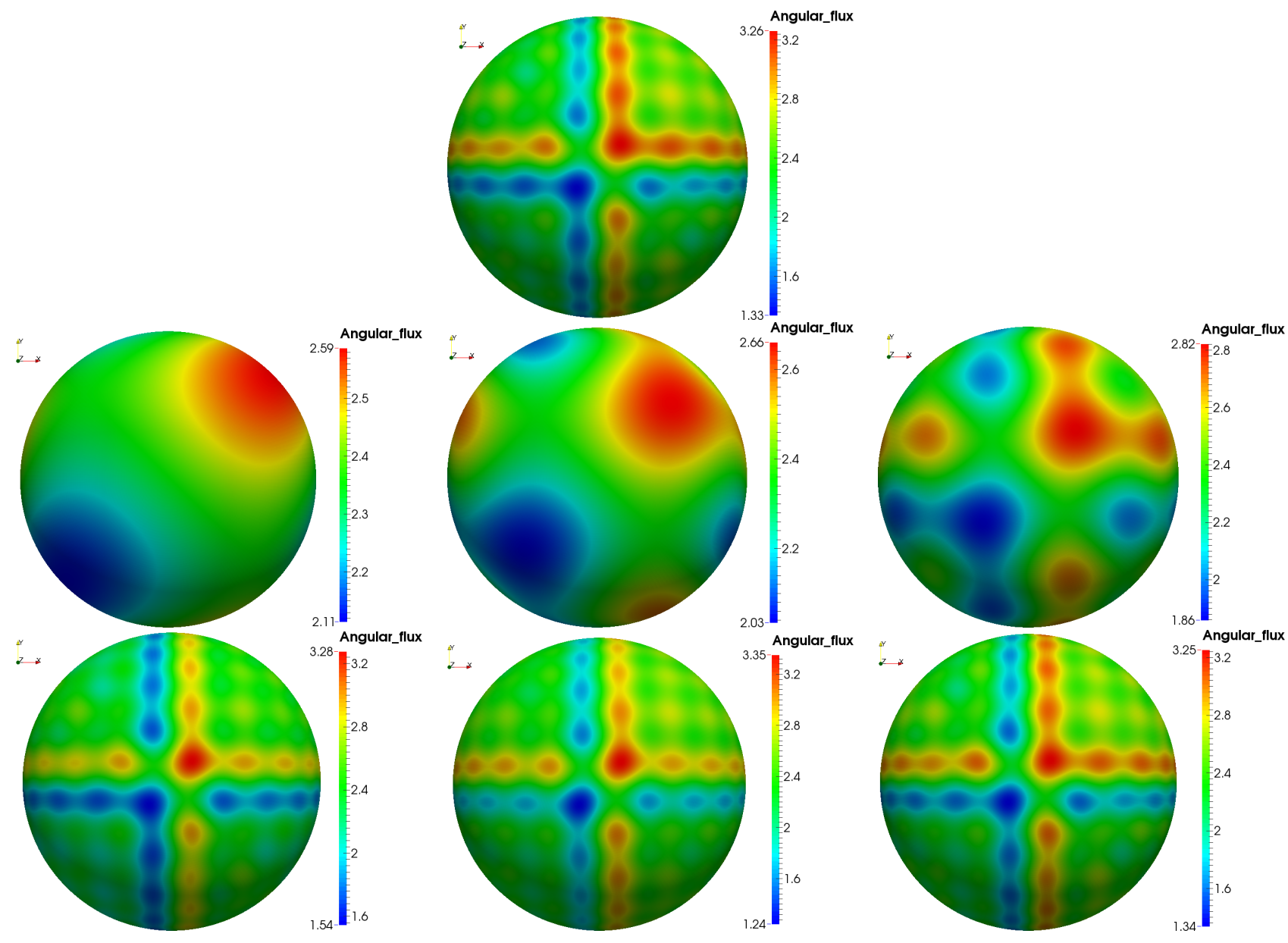

Figure 19: Angular flux profiles at position A of the Adam's pincell problem. Top: exact solution, Middle: $P_{3}, P_{5}, P_{9}$ solutions, Bottom $P O D_{10}, P O D_{21}, P O D_{55}$ solutions. 

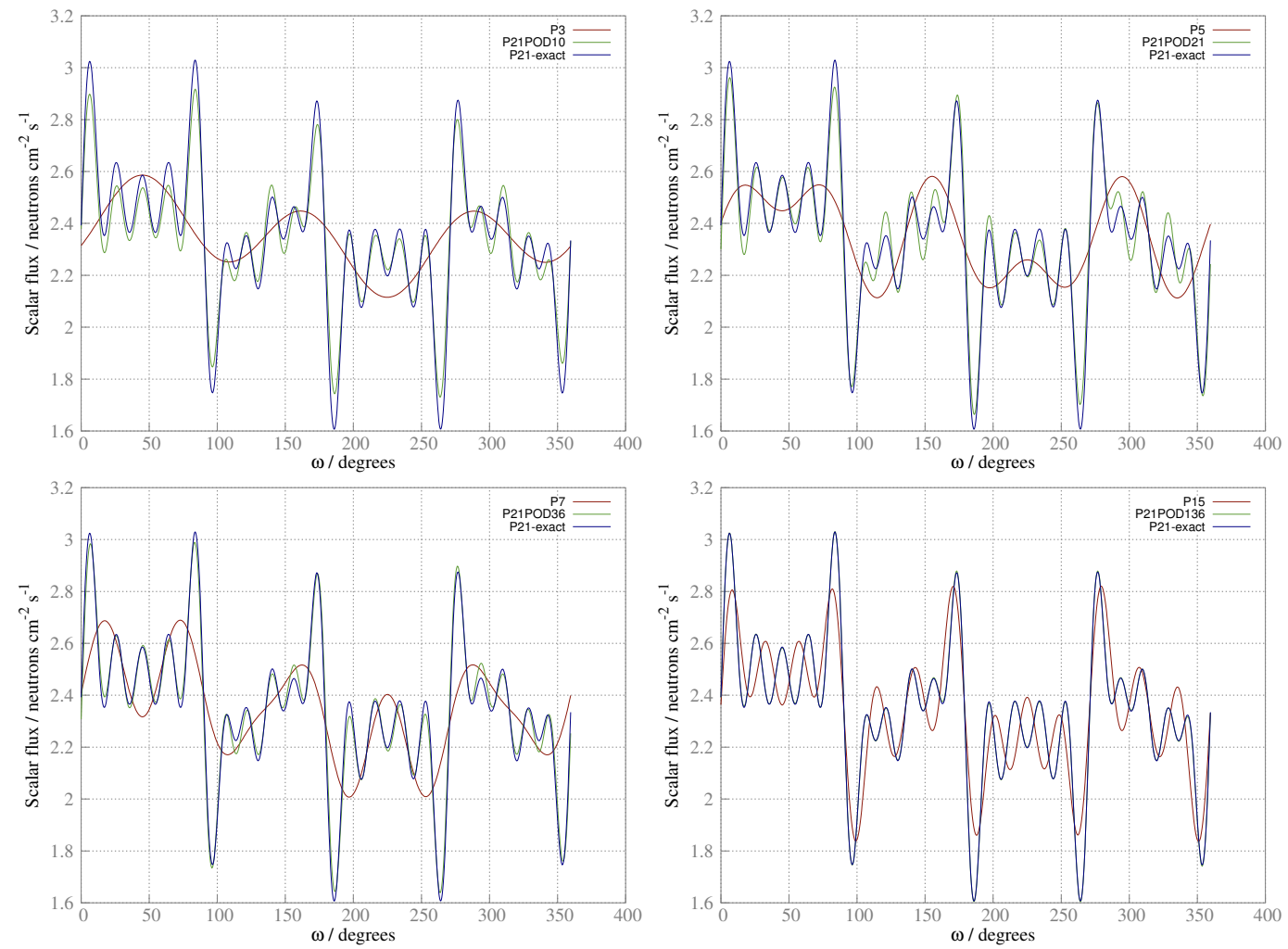

Figure 20: Angular flux profile through the azimuthal direction $\omega, \mu=0.33$, at position A of the Adam's pincell problem. Left to right, top to bottom: $P_{3} \& P O D_{10}$ solutions (10 basis functions), $P_{5} \& P O D_{21}$ solutions (21 basis functions), $P_{7} \& P_{36}$ solutions (36 basis functions), $P_{15} \& P O D_{136}$ solutions (55 basis functions).

A final point worthy of mentioning is with regards to the options of relieving the computational expense when solving the full model in order to record the snapshots. Full model simulations can be very expensive, especially for large scale reactor physics problems, and this could make generating the POD functions computationally infeasible. However there are possible routes that can remove this burden, one of which would be to solve the full problem on a reduced sized mesh. Provided that there is still sufficient resolution to capture the angular distributions, the POD model can be generated more efficiently and then used to solve the problem on a refined, and much larger, spatially converged mesh. Alternatively a reactor physics problem can be broken down into smaller sub problems that can be solved efficiently. For example, a single fuel pin with reflective boundary conditions will provide good representations of the neutron distribution within all pins throughout the central parts of the core. These solutions are relatively cheap to compute using a full model, but the resulting POD model can then be used to model the entirety of the core. These options, amongst others, will be the focus of 

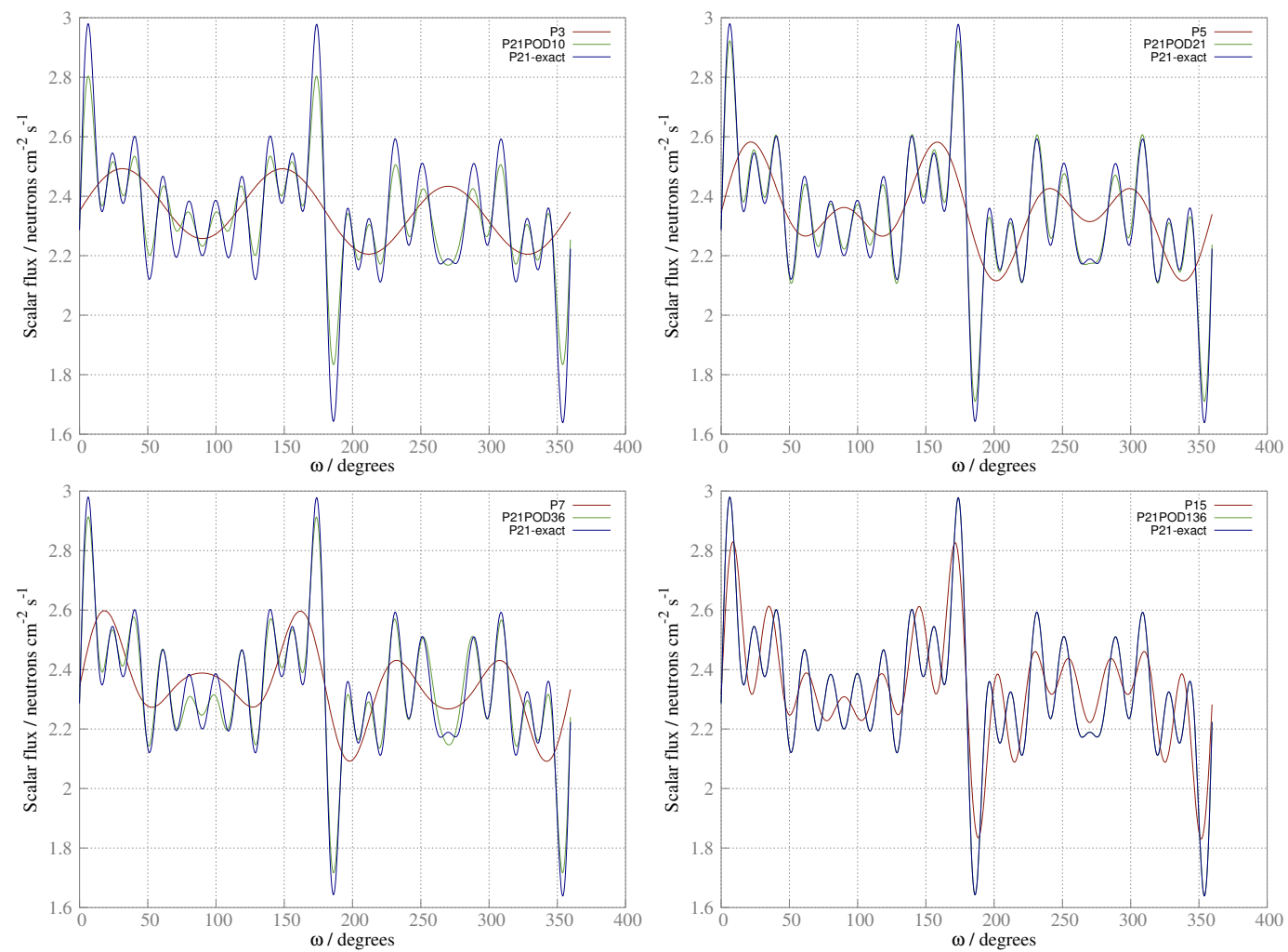

Figure 21: Angular flux profile through the azimuthal direction $\omega, \mu=0.33$, at position B of the Adam's pincell problem. Left to right, top to bottom: $P_{3} \& P O D_{10}$ solutions (10 basis functions), $P_{5} \& P O D_{21}$ solutions (21 basis functions), $P_{7} \& P O D_{36}$ solutions (36 basis functions), $P_{15} \& P O D_{136}$ solutions (55 basis functions). 


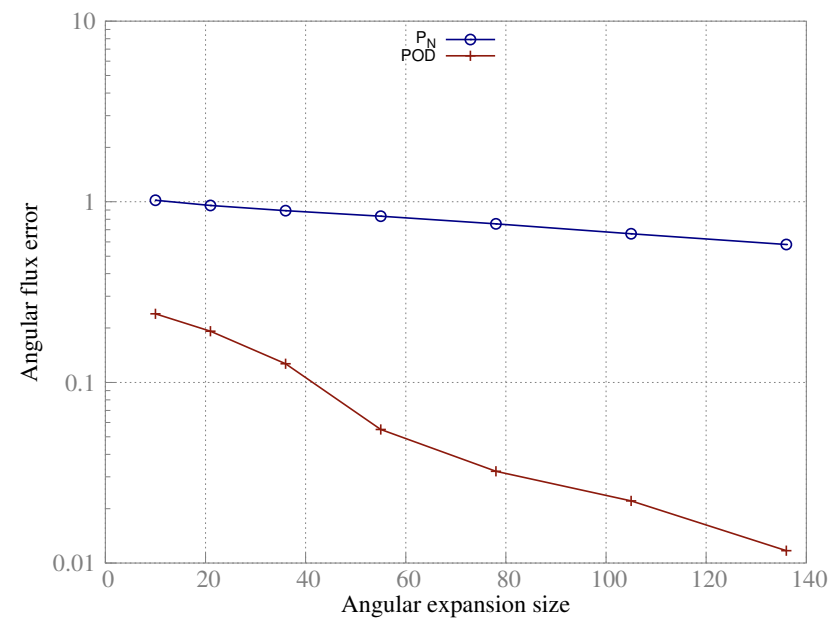

Figure 22: Error norms for $P_{N}$ and POD for the angular flux solutions at node 9795.

investigation in subsequent papers.

\section{ACKNOWLEDGEMENTS}

We acknowledge the funding of this work through the EPSRC grant ref: EP/M022684/1 and EP/J002011/1. Prof. I.M. Navon acknowledges the support of NSF grant ATM-0931198.

[1] K. Fukunaga. Introduction to Statistical Pattern Recognition. Computer Science and Scientific Computing. Academic Press, second edition, 1990.

[2] I. T. Jolliffe. Principal Component Analysis. Springer, Berlin, second edition, October 2002.

[3] D.T. Crommelin and A.J. Majda. Strategies for model reduction: comparing different optimal bases. Journal of the Atmospheric Sciences, 61:2206-2217, 2004.

[4] A. J. Majda, I. Timofeyev, and E. Vanden-Eijnden. Systematic Strategies for Stochastic Mode Reduction in Climate. Journal of the Atmospheric Sciences, 60(14):1705-1722, July 2003.

[5] J. Du, J Zhu, and I. M. Navon. An optimizing finite difference scheme based on proper orthogonal decomposition for CVD equations. International Journal for Numerical Methods in Biomedical Engineering, 27:78-94, 2011. 
[6] Y. Cao, I.M. Navon, and Z. Luo. A reduced order approach to four-dimensional variational data assimilation using proper orthogonal decomposition. International Journal for Numerical Methods in Fluids, 53:1571-1583, 2007.

[7] Y. Cao, J. Zhu, Z. Luo, and I.M. Navon. Reduced order modeling of the upper tropical pacific ocean model using proper orthogonal decomposition. Computers $\&$ Mathematics with Applications, 52:1373-1386, 2006.

[8] L. Sirovich. Turbulence and the dynamics of coherent structures. Quarterly of Applied Mathematicss, 5:561 - 590, 1987.

[9] L. Sirovich. Turbulence and the dynamics of coherent structures, part III: Dynamics and scaling. Quarterly of Applied Mathematics, XLV:583-590, 1987.

[10] K. Pearson. On lines and planes of closest fit to systems of points in space. Philosophical Magazine, 2(6):559-572, 1901.

[11] J. L. Lumley. The Structure of Inhomogeneous Turbulent Flows. In Atmospheric turbulence and radio propagation, pages 166-178. Nauka, Moscow, 1967.

[12] H. P. Bakewell and J. L. Lumley. Viscous sublayer and adjacent wall region in turbulent pipe flow. The Physics of Fluids, 10:1880-1889, 1967.

[13] F.R. Payne and J.L. Lumley. Large-eddy structure of the turbulent wake behind a circular cylinder. The Physics of Fluids, 10:194-196, 1967.

[14] A. E. Dean and C. Mavriplis. Low-dimensional description of the dynamics in separated flow past thick airfoil. The American Institute of Aeronautics and Astronautics, 32:1222-1227, 1994.

[15] K. S. Ball, L. Sirovich, and L. R. Keefe. Dynamical eigenfunction decomposition of turbulent channel flow. International Journal for Numerical Methods in Fluids, 12(6):585-604, 1991.

[16] J. Delville, S. Bellin, and J. P. Bonnet. Use of the proper orthogonal decomposition in a plane turbulent mixing layer, in Turbulence and Coherent Structures; Selected Papers from Turbulence 89, pages 75-90. Kluwer Academic Publishers, Dordrecht, 1991.

[17] L. Sirovich and H. Park. Turbulent thermal convection in a finite domain: PartI. theory. Physics of fluids, 2:1649-1658, 1990.

[18] H. Park and L. Sirovich. Turbulent thermal convection in a finite domain: PartII. numerical results. Physics of fluids, 2:1659-1668, 1990.

[19] F. Fang, C.C. Pain, I.M. Navon, G.J. Gorman, M.D. Piggott, P.A. Allison, P.E. Farrell, and A.J.H. Goddard. A POD reduced order unstructured mesh ocean modelling method for moderate reynolds number flows. Ocean Modelling, 28:127 - 136, 2009. 
[20] R. Stefanescu and I.M. Navon. POD/DEIM Nonlinear model order reduction of an adi implicit shallow water equations model. Journal of Computational Physics, 237:95-114, 2013.

[21] C. L. Pettit and P. S. Beran. Application of proper orthogonal decomposition to the discrete euler equations. International Journal for Numerical Methods in Engineering, 55(4):479-497, 2002.

[22] J. Burkardt, M. Gunzburger, and H. Lee. POD and CVT-based reduced-order modeling of Navier-Stokes flows. Computer Methods in Applied Mechanics and Engineering, 196:337-355, 2006.

[23] J. Du, I.M. Navon, J.L. Steward, A.K. Alekseev, and Z. Luo. Reduced-order modeling based on POD of a parabolized Navier-Stokes equation model I: forward model. International Journal for Numerical Methods in Fluids, 69(3):710-730, 2012.

[24] J. Du, I.M. Navon, A.K. Alekseev, and Z. Luo. Reduced-order modeling based on POD of a parabolized Navier-Stokes equation model II: Trust region POD 4-D VAR data assimilation. Computers 85 Mathematics with Applications, 65:380-394, 2013.

[25] S. Chaturantabut and D.C. Sorensen. Nonlinear model reduction via discrete empirical interpolation. SIAM Journal of Scientific Computing, 32:2737-2764, 2010.

[26] J. Du, F. Fang, C.C. Pain, I.M. Navon, J. Zhu, and D.A. Ham. POD reduced-order unstructured mesh modeling applied to 2D and 3D fluid flow. Computers and Mathematics with Applications, 65:362-379, 2013.

[27] D. Xiao, F. Fang, A.G. Buchan, C.C. Pain, I.M. Navon, J. Du, and G. Hu. Non-linear model reduction for the Navier-Stokes equations using residual DEIM method. Journal of Computational Physics, 263(0):1 - 18, 2014.

[28] F. Wols. Transient analyses of accelerator driven systems using modal expansion techniques. Master's thesis, Delft University of Technology, 2010.

[29] A. Sartori, D. Baroli, A. Cammi, D. Chiesa, L. Luzzi, R. Ponciroli, E. Previtali, M. E. Ricotti, G. Rozza, and M. Sisti. Comparison of a modal method and a proper orthogonal decomposition approach for multi-group time-dependent reactor spatial kinetics. Annals of Nuclear Energy, 71(0):217 - 229, 2014.

[30] A. G. Buchan, C. C. Pain, F. Fang, and I. M. Navon. A POD reduced-order model for eigenvalue problems with application to reactor physics. International Journal for Numerical Methods in Engineering, 95(12):1011-1032, 2013.

[31] Richard L. Reed and Jeremy A. Roberts. An energy basis for response matrix methods based on the Karhunen-Loéve transform. Annals of Nuclear Energy, 78(0):70 - 80, 2015. 
[32] A. G. Buchan, C. C.Pain, M. D. Eaton, R. P. Smedley-Stevenson, and A. J. H. Goddard. Linear and quadratic octahedral wavelets on the sphere for angular discretisations of the Boltzmann transport equation. Annals of Nuclear Energy, 32:1224-1273, 2005.

[33] A. G. Buchan, A. S. Candy, S. R. Merton, C. C.Pain, J. L. Hadi, M. D.Eaton, A. J. H. Goddard, R. P. Smedley-Stevenson, and G. J. Pearce. The inner-element subgrid scale finite element method for the Boltzmann transport equation. Nuclear Science and Engineering, 164:105-122, 2010.

[34] A. G. Buchan, C. C. Pain, A. P. Umpleby, and R. P. Smedley-Stevenson. A sub-grid scale finite element agglomeration multigrid method with application to the Boltzmann transport equation. International Journal for Numerical Methods in Engineering, 92(3):318-342, 2012.

[35] M. D. Eaton. A High-Resolution Riemann Method for Solving Radiation Transport Problems on Unstructured Meshes. PhD thesis, Imperial College of Science, Technology and Medicine, University of London, 2004.

[36] A. G. Buchan and C. C.Pain. An efficient space-angle subgrid scale discretisation of the neutron transport equation. submitted to Annals of Nuclear Energy, 2014.

[37] M. L. Adams. Angular Dependence of the Fast Flux in Reactor Lattices. In American Nuclear Society 2001 Annual Meeting, pages 212-214, 2001.

[38] S. Balay, K. Buschelman, V. Eijkhout, W. D. Gropp, D. Kaushik, M. G. Knepley, L. C. McInnes, B. F. Smith, and H. Zhang. Portable, extensible toolkit for scientific computation (PETSc) users manual. Technical Report ANL-95/11 - Revision 2.1.5, Argonne National Laboratory, 2004.

[39] M. A. Goffin, A. G. Buchan, A. C. Belme, C. C. Pain, M. D. Eaton, P. N. Smith, and R. P. Smedley-Stevenson. Goal-based angular adaptivity applied to the spherical harmonics discretisation of the neutral particle transport equation. Annals of Nuclear Energy, 71:60-80, September 2014.

[40] K. Manalo, C. D. Ahrens, and G. Sjoden. Advanced quadratures for three-dimensional discrete ordinate transport simulations: A comparative study. Annals of Nuclear Energy, 81(0):196 - 206, 2015 . 